\title{
Instantons in QCD with Many Colors
}

\author{
T. Schäfer ${ }^{1,2,3}$ \\ ${ }^{1}$ Department of Physics, Duke University, Durham, NC 27708 \\ ${ }^{2}$ Department of Physics, SUNY Stony Brook, Stony Brook, NY 11794 \\ ${ }^{3}$ Riken-BNL Research Center, Brookhaven National Laboratory, Upton, NY 11973
}

\begin{abstract}
We study instantons in QCD with many colors. We first discuss a number of qualitative arguments concerning the large $N_{c}$ scaling behavior of a random instanton ensemble. We show that most hadronic observables are compatible with standard large $N_{c}$ counting rules provided the average instanton size is $O(1)$ and the instanton density is $O\left(N_{c}\right)$ in the large $N_{c}$ limit. This is not the case for the topological susceptibility and the mass of the $\eta^{\prime}$. For these observables consistency with conventional large $N_{c}$ counting requires that fluctuations in the instanton liquid are suppressed compared to Poissonian fluctuations. Using mean field estimates and numerical simulations we show that the required scaling behavior of the instanton density is natural in models in which the instanton density is regularized in terms of a classical repulsive core. We also show that in these models fluctuations of the topological charge are suppressed and that $m_{\eta^{\prime}}^{2}=O\left(1 / N_{c}\right)$. We conclude that the instanton liquid model is not necessarily in conflict with the $1 / N_{c}$ expansion.
\end{abstract}




\section{INTRODUCTION}

Quantum Chromodynamics (QCD), the theory of the strong interactions, is an essentially parameter free theory. The QCD lagrangian contains a coupling constant, $g$, but because of the phenomenon of dimensional transmutation this parameter is replaced by a dimensionful scale parameter, $\Lambda_{Q C D}$. As a consequence, there is no obvious expansion parameter in QCD. 't Hooft proposed to use the number of colors, $N_{c}$, as a parameter [1]. He suggested that QCD simplifies in the limit $N_{c} \rightarrow \infty$ and that $1 / N_{c}$ can be treated as an expansion parameter.

We do not know how to solve QCD in the large $N_{c}$ limit. However, by analyzing the $N_{c}$ dependence of classes of Feynman diagrams we can make certain qualitative statements about the structure of large $N_{c}$ QCD. We expect, for example, that the masses of mesons and glueballs are $O(1)$ whereas the masses of baryons are $O\left(N_{c}\right)$. Also, meson decay constants are $O\left(N_{c}^{1 / 2}\right)$ and meson-meson scattering amplitudes are $O\left(1 / N_{c}\right)$. As a result, large $N_{c}$ QCD is a theory of weakly interacting mesons and glueballs.

The fate of the $U(1)_{A}$ anomaly in the large $N_{c}$ limit is an interesting question. The problem is complicated by the fact that if the $\theta$ term,

$$
\mathcal{L}=\frac{i g^{2} \theta}{32 \pi^{2}} G_{\mu \nu}^{a} \tilde{G}_{\mu \nu}^{a}
$$

is added to the QCD lagrangian then in perturbation theory there is no dependence on the parameter $\theta$. Witten suggested that non-perturbative effects generate $\theta$-dependence in the pure gauge theory and that the topological susceptibility,

$$
\chi_{\text {top }}=\left.\frac{d^{2} E}{d \theta^{2}}\right|_{\theta=0},
$$

is $O(1)$ in the large $N_{c}$ limit [2, 3]. Standard large $N_{c}$ counting suggests that the contribution of fermions to $\chi_{\text {top }}$ is subleading in $1 / N_{c}$. We know, however, that this is not correct, because there is no $\theta$-dependence in QCD with massless fermions. Witten argued that this apparent contradiction can be resolved if the mass of the $\eta^{\prime}$ meson scales as $N_{c}^{-1 / 2}$ in the large $N_{c}$ limit. Witten [4] and Veneziano [5] derived a relation between the mass of the $\eta^{\prime}$ and the topological susceptibility in pure gauge theory,

$$
\frac{f_{\pi}^{2}}{2 N_{f}} m_{\eta^{\prime}}^{2}=\chi_{t o p} .
$$


Using $\chi_{\text {top }}=O(1)$ and $f_{\pi}^{2}=O\left(N_{c}\right)$ we observe that indeed $m_{\eta^{\prime}}^{2}=O\left(1 / N_{c}\right)$. This result implies that the $U(1)_{A}$ anomaly is effectively restored in the large $N_{c}$ limit.

The $\theta$-dependence of vacuum energy is related to topological properties of QCD. In the semi-classical approximation these features can be described in terms of instantons. Instantons are localized field configurations that carry topological charge [6]

$$
Q_{\text {top }}=\frac{g^{2}}{32 \pi^{2}} \int d^{4} x G_{\mu \nu}^{a} \tilde{G}_{\mu \nu}^{a}= \pm 1 .
$$

In 1978 Witten observed that classical effects, such as instantons, scale as $\exp \left(-1 / g^{2}\right) \sim$ $\exp \left(-N_{c}\right)$ which seems to contradict the assumption $\chi_{\text {top }}=O(1)$ [2]. On the other hand, it was also found that the assumption that topological fluctuations are semi-classical leads to a very successful picture of the QCD vacuum, termed the instanton liquid model [7, 8, 9. 10]. The instanton liquid model postulates that for $N_{c}=3$ the density of instantons is approximately $(N / V) \simeq 1 \mathrm{fm}^{-4}$ while the average size is $\rho \simeq 1 / 3 \mathrm{fm}$. These numbers reproduce the topological susceptibility in the pure gauge theory $\chi_{\text {top }} \simeq(200 \mathrm{MeV})^{4}$ and the chiral condensate $\langle\bar{\psi} \psi\rangle \simeq-(230 \mathrm{MeV})^{3}$. More detailed calculations show that the instanton liquid model successfully describes an impressive amount of data on hadronic correlation functions [10, 11, 12].

Topological properties of the QCD vacuum have also been studied in lattice QCD. It was found that the topological susceptibility in pure gauge QCD is $\chi_{\text {top }} \simeq(200 \mathrm{MeV})^{4}$ [13], as predicted by the Witten-Veneziano relation equ. (3)). However, it was also observed that the topological susceptibility is very stable under cooling, and appears to be dominated by semi-classical configurations. Lattice simulations also seem to confirm the values of the key parameters of the instanton liquid [14], $(N / V) \simeq 1 \mathrm{fm}^{-4}$ and $\rho \simeq 1 / 3 \mathrm{fm}$. Recent work has focused on the relation of instantons and low-lying eigenstates of the Dirac operator. The instanton model predicts that the lowest eigenstates of the Dirac operator, which dominate chiral symmetry breaking, are linear combinations of localized, approximately chiral states associated with the fermionic zero modes of individual instantons and anti-instantons [15]. This picture has been confirmed by lattice calculations [16, 17].

In light of these results the question arises whether the success of the instanton model can be reconciled with the large $N_{c}$ expansion. In order to address this problem we have investigated the predictions of the instanton model for QCD with many colors. This paper is organized as follows. In Sect. [1] we present qualitative arguments based on a random 
instanton liquid. In Sects. $\llbracket 1 \mathrm{I}$ and $\mathbb{\mathrm { IV }}$ we discuss analytic results obtained in the mean field approximation. In Sects. $\nabla$ and $\nabla \mathbb{1}$ we discuss the results of numerical simulations of the interacting instanton liquid.

\section{RANDOM INSTANTON LIQUID}

The simplest picture of the instanton liquid in QCD is the random instanton liquid model proposed by Shuryak [8]. This model is based on the assumption that the instanton ensemble is characterized by two key parameters, the instanton density $(N / V)$ and the typical instanton size $\rho$. Except for the size, the collective coordinates of the instantons are distributed randomly. The model refers to an instanton liquid rather than an instanton gas because interactions between instanton cannot be ignored completely. This is particularly clear if massless fermions are present. If interactions are ignored then every instanton contributes an exact zero mode to the spectrum of the Dirac operator and the total density of instantons is exactly zero. Interactions lead to mixing between the zero modes and qualitatively change the spectrum of the Dirac operator. As a consequence, chiral symmetry is spontaneously broken and the density of instantons is non-zero.

In order to understand the large $N_{c}$ behavior of the instanton liquid we have to understand the scaling behavior of the two parameters introduced above. In this paper we shall argue that

$$
\frac{N}{V}=O\left(N_{c}\right), \quad \rho=O(1) .
$$

In section [1] we will show that equ. (5) follows from the partition function of the instanton liquid if the instanton size distribution is regularized by a classical repulsive core. Before we discuss this result we would like to provide some plausibility arguments and explore simple consequences of the scaling relations.

We first consider the average instanton size $\rho$. At one-loop order the instanton action is given by $S_{0}=\left(8 \pi^{2}\right) / g^{2}=-b \log (\rho \Lambda)$ where $b=\left(11 N_{c}\right) / 3$ is the first coefficient of the beta function in pure gauge QCD. In the 't Hooft limit $N_{c} \rightarrow \infty$ with $g^{2} N_{c}=$ const we

expect $S_{0}=O\left(N_{c}\right)$ and $\rho=O(1)$. We have to keep in mind, however, that instantons in QCD come in all sizes. In the semi-classical approximation we cannot study instantons with size $\rho \sim \Lambda^{-1}$ and action $S_{0} \sim 1$. There is evidence, however, that chiral symmetry breaking in QCD with $N_{c}=3$ colors is dominated by small instantons. A priori it is not 
clear whether this remains true in the large $N_{c}$ limit. Our strategy in this work is to assume that the semi-classical approximation remains valid and to show that this assumption leads to a consistent picture.

In the random instanton liquid model the instanton density is related to the nonperturbative gluon condensate

$$
\frac{N}{V}=\frac{1}{32 \pi^{2}}\left\langle g^{2} G_{\mu \nu}^{a} G_{\mu \nu}^{a}\right\rangle
$$

Standard $N_{c}$ counting suggests that $\left\langle g^{2} G^{2}\right\rangle=O\left(N_{c}\right)$ and we are lead to the conclusion that $(N / V)=O\left(N_{c}\right)$. This is also consistent with the expected scaling of the vacuum energy. Using equ. (6) and the trace anomaly relation

$$
\left\langle T_{\mu \mu}\right\rangle=-\frac{b}{32 \pi^{2}}\left\langle g^{2} G_{\mu \nu}^{a} G_{\mu \nu}^{a}\right\rangle,
$$

the vacuum energy density is given by

$$
\epsilon=-\frac{b}{4}\left(\frac{N}{V}\right)
$$

Using $(N / V)=O\left(N_{c}\right)$ we find that the vacuum energy scales as $\epsilon=O\left(N_{c}^{2}\right)$ which agrees with our expectations for a system with $N_{c}^{2}$ gluonic degrees of freedom. We should note that equ. (8) requires interactions between instantons. The partition function of a completely non-interacting gas of instantons gives $\epsilon \sim(N / V) \sim N_{c}$.

We also note that $(N / V)=O\left(N_{c}\right)$ implies that the effective "packing fraction" of instantons remains constant in the large $N_{c}$ limit. Instantons with topological charge $Q_{\text {top }}= \pm 1$ in $S U\left(N_{c}\right)$ QCD are embeddings of $S U(2)$ instantons. Since the number of mutually commuting $S U(2)$ subgroups of $S U\left(N_{c}\right)$ scales as $N_{c}$ we can have $O\left(N_{c}\right)$ instantons which overlap in space but are in fact weakly interacting. Witten argued that the only alternatives are $S_{0}=O\left(N_{c}\right)$ and $(N / V)=O\left(e^{-N_{c}}\right)$ or $S_{0}=O(1)$ and $(N / V)=O\left(e^{N_{c}}\right)$. However, as we shall see in the next section, it is possible for the instanton density to remain finite even if $S_{0}=O\left(N_{c}\right)$ because the large entropy of instantons in $S U\left(N_{c}\right)$ can overcome the exponential suppression due to the action.

If instantons are distributed randomly then fluctuations in the number of instantons and anti-instantons are expected to be Poissonian. This leads to the predictions

$$
\begin{aligned}
\left\langle N^{2}\right\rangle-\langle N\rangle^{2} & =\langle N\rangle, \\
\left\langle Q^{2}\right\rangle & =\langle N\rangle
\end{aligned}
$$


where $N=N_{I}+N_{A}$ is the total number of instantons and $Q=N_{I}-N_{A}$ is the topological charge. Equ. (10) implies that

$$
\chi_{\text {top }}=\frac{\left\langle Q^{2}\right\rangle}{V}=\left(\frac{N}{V}\right) .
$$

Using $(N / V)=O\left(N_{c}\right)$ we observe that $\chi_{t o p}=O\left(N_{c}\right)$ which is in contradiction to Witten's assumption $\chi_{\text {top }}=O(1)$. However, as we shall see in the next section, interactions between instantons cannot be ignored in the large $N_{c}$ limit and the fluctuations are suppressed compared to equs. (9,10).

Finally, we would like to study chiral symmetry breaking in a random instanton liquid. For definiteness, we will consider the case $N_{f}=2$ but the conclusions are of course independent of the number of flavors. Instantons induce an effective $2 N_{f}$-fermion lagrangian. After averaging over the color orientation of the instanton the effective lagrangian is given by 18,19

$$
\begin{gathered}
\mathcal{L}=\int n(\rho) d \rho \frac{2(2 \pi \rho)^{4} \rho^{2}}{4\left(N_{c}^{2}-1\right)} \epsilon_{f_{1} f_{2}} \epsilon_{g_{1} g_{2}}\left(\frac{2 N_{c}-1}{2 N_{c}}\left(\bar{\psi}_{L, f_{1}} \psi_{R, g_{1}}\right)\left(\bar{\psi}_{L, f_{2}} \psi_{R, g_{2}}\right)\right. \\
\left.-\frac{1}{8 N_{c}}\left(\bar{\psi}_{L, f_{1}} \sigma_{\mu \nu} \psi_{R, g_{1}}\right)\left(\bar{\psi}_{L, f_{2}} \sigma_{\mu \nu} \psi_{R, g_{2}}\right)+(L \leftrightarrow R)\right) .
\end{gathered}
$$

We observe that the explicit $N_{c}$ dependence is given by $1 / N_{c}^{2}$. This is again related to the fact that instantons are $S U(2)$ objects. Quarks can only interact via instanton zero modes if they overlap with the color wave function of the instanton. As a result, the probability that two quarks with arbitrary color propagating in the background field of an instanton interact is $O\left(1 / N_{c}^{2}\right)$.

Chiral symmetry breaking can be studied in the mean field approximation. We will address this problem in much more detail in the following section but we can give a simple qualitative argument here. In the mean field approximation we can derive a gap equation for the spontaneously generated constituent quark mass. The gap equation is of the form

$$
M=G N_{c} \int \frac{d^{4} k}{(2 \pi)^{4}} \frac{M}{M^{2}+k^{2}},
$$

where $M$ is the constituent mass and $G$ is the effective coupling constant in equ. (12). The factor $N_{c}$ comes from doing the trace over the quark propagator. The coupling constant $G$ scales as $1 / N_{c}$ because the density of instantons is $O\left(N_{c}\right)$ and the effective lagrangian contains an explicit factor $1 / N_{c}^{2}$. We conclude that the coefficient in the gap equation is $O(1)$ and that the dynamically generated quark mass is $O(1)$ also. This also implies that the quark condensate, which involves an extra sum over color, is $O\left(N_{c}\right)$. 


\section{THE MEAN FIELD APPROXIMATION}

In this section we shall make the arguments presented in the previous section more quantitative. Instead of considering the average instanton size and instanton size density to be arbitrary parameters, we shall determine these quantities from the partition function of the instanton ensemble. This means, in particular, that both $\rho$ and $(N / V)$ are expressed in terms of the fundamental scale parameter of QCD. For this purpose it is essential to take the interaction between instantons into account. If instantons are semi-classical, $S_{\text {inst }} \gg 1$, and if the interaction between instantons is weak, $S_{i n t} \ll S_{\text {inst }}$, this can be accomplished using mean field methods [9, 20, 21]. In this section, we shall follow the variational method of Diakonov and Petrov [9].

We consider the partition function for a system of instantons in pure gauge theory

$$
Z=\frac{1}{N_{I} ! N_{A} !} \prod_{I}^{N_{I}+N_{A}} \int\left[d \Omega_{I} n\left(\rho_{I}\right)\right] \exp \left(-S_{i n t}\right) .
$$

Here, $\Omega_{I}=\left(z_{I}, \rho_{I}, U_{I}\right)$ are the collective coordinates of the instanton $I$ and $n(\rho)$ is the semi-classical instanton distribution function 22]

$$
\begin{aligned}
n(\rho)= & C_{N_{c}}\left(\frac{8 \pi^{2}}{g^{2}}\right)^{2 N_{c}} \rho^{-5} \exp \left[-\frac{8 \pi^{2}}{g(\rho)^{2}}\right], \\
C_{N_{c}} & =\frac{0.466 \exp \left(-1.679 N_{c}\right)}{\left(N_{c}-1\right) !\left(N_{c}-2\right) !}, \\
\frac{8 \pi^{2}}{g^{2}(\rho)} & =-b \log (\rho \Lambda), \quad b=\frac{11}{3} N_{c} .
\end{aligned}
$$

We have denoted the classical instanton interaction by $S_{i n t}$. If the instanton ensemble is sufficiently dilute we can approximate the instanton interaction as a sum of two-body terms, $S_{i n t}=\sum_{I J} S_{I J}$. For a well separated instanton-anti-instanton pair the interaction has the dipole structure [7]

$$
S_{\text {int }}=-\frac{32 \pi^{2}}{g^{2}} \frac{\rho_{I}^{2} \rho_{A}^{2}}{R_{I A}^{4}}|u|^{2}\left(1-4 \cos ^{2} \theta\right) .
$$

Here $\rho_{I, A}$ are instanton radii and $R_{I A}$ is the instanton-anti-instanton separation. The relative color orientation is characterized by a complex four-vector $u_{\mu}=\frac{1}{2 i} \operatorname{tr}\left(U_{I A} \tau_{\mu}^{+}\right)$, where $U_{I A}=$ $U_{I} U_{A}^{\dagger}$ depends on the rigid gauge transformations that describe the color orientation of the individual instanton and anti-instanton and $\tau_{\mu}^{+}=(\vec{\tau},-i)$. We have also defined the relative color angle $\cos ^{2} \theta=|u \cdot \hat{R}|^{2} /|u|^{2}$. The dipole interaction is valid if $R_{I A}^{2} \gg \rho_{I} \rho_{A}$. We will specify the interaction at shorter distances below. 
Diakonov and Petrov suggested to analyze the partition function equ. (14) using a variational single-instanton distribution $\mu(\rho)$ [9]. The corresponding partition function is

$$
Z_{1}=\frac{1}{N_{I} ! N_{A} !} \prod_{i}^{N_{I}+N_{A}} \int d \Omega_{I} \mu\left(\rho_{I}\right)=\frac{1}{N_{I} ! N_{A} !}\left(V \mu_{0}\right)^{N_{I}+N_{A}}
$$

where $\mu_{0}=\int d \rho \mu(\rho)$. The exact partition function is

$$
Z=Z_{1}\left\langle\exp \left(-\left(S-S_{1}\right)\right)\right\rangle
$$

where $S$ is the full action, $S_{1}=\log (\mu(\rho))$ is the variational estimate and the average $\langle$.$\rangle is$ computed using the variational distribution function. The partition function satisfies the bound

$$
Z \geq Z_{1} \exp \left(-\left\langle S-S_{1}\right\rangle\right)
$$

which follows from convexity. The optimal distribution function $\mu(\rho)$ is determined from a variational principle, $(\delta \log Z) /(\delta \mu(\rho))=0$, where $Z$ is computed from equ. (21). One can show that the variational result for the free energy $F=-\log (Z) / V$ provides an upper bound on the true free energy.

The calculation of $\left\langle S-S_{1}\right\rangle$ reduces to the calculation of the average instanton interaction $\left\langle S_{\text {int }}\right\rangle$. Since the variational ansatz does not include any correlations, we only need to average the instanton interaction over the collective coordinates of the two instantons. The dipole interaction (18) vanishes when averaged over all color orientations. In [9] it was proposed to compute the instanton interaction at all distances using a specific ansatz (called the "sum ansatz") for the two-instanton configuration. The result is that both the instanton-instanton $(I I)$ and instanton-anti-instanton $(I A)$ are repulsive on the average. We find

$$
\left\langle S_{\text {int }}\right\rangle=\frac{8 \pi^{2}}{g^{2}} \gamma^{2} \rho_{I}^{2} \rho_{J}^{2}, \quad \gamma^{2}=\frac{27}{4} \frac{N_{c}}{N_{c}^{2}-1} \pi^{2} .
$$

The interaction contains an explicit factor $N_{c} /\left(N_{c}^{2}-1\right) \sim 1 / N_{c}$ which reflects the probability that two random instantons overlap in color space. Since the classical action scales as $S_{0} \sim 1 / g^{2}$ we find that the average interaction between any two instantons is $O(1)$. Applying the variational principle, one finds [9]

$$
\mu(\rho)=n(\rho) \exp \left[-\beta \gamma^{2}\left(\frac{N \overline{\rho^{2}}}{V}\right) \rho^{2}\right],
$$

where $\beta=\beta(\bar{\rho})$ is the average instanton action and $\overline{\rho^{2}}$ is the average size. We observe that the single instanton distribution is cut off at large sizes by the average instanton repulsion. 
The instanton density and average size are given by

$$
\begin{aligned}
& \frac{N}{V}=\Lambda^{4}\left[C_{N_{c}} \beta^{2 N_{c}} \Gamma(\nu)\left(\beta \nu \gamma^{2}\right)^{-\nu / 2}\right]^{\frac{2}{2+\nu}}, \\
& \overline{\rho^{2}}=\left(\frac{\nu V}{\beta \gamma^{2} N}\right)^{1 / 2}, \quad \nu=\frac{b-4}{2}
\end{aligned}
$$

We note that $\Lambda$ is the only dimensionful parameter. The free energy is given by

$$
F=-\frac{b}{4}\left(\frac{N}{V}\right)
$$

which is in agreement with the trace anomaly. We can now study the dependence of $(N / V)$ and $\rho$ on $N_{c}$, see Fig. 1. We note that to one-loop order the scale in the pre-exponent $\beta(\bar{\rho})$ is not well determined. In practice we assume that $\beta=N_{c} s_{0}$ with $s_{0}=5$. Changing $s_{0}$ does affect both $(N / V)$ and $\rho$ but the main effect can be absorbed in the scale parameter. The remaining dependence on $s_{0}$ is very weak.

Fig. 11 shows that for $N_{c}>4$ the average instanton size is essentially constant while the instanton density grows linearly with $N_{c}$. This is easily verified by inspecting equ. (24). Expanding $\log (N / V)$ in powers of $N_{c}$ and $\log \left(N_{c}\right)$ we observe that independent of the details of the interaction the instanton density scales at most as a power, not an exponential, in $N_{c}$ [23]. Using the fact that $\gamma^{2}=O\left(1 / N_{c}\right)$, which is equivalent to $\left\langle S_{\text {int }}\right\rangle=O(1)$, we find that $(N / V)=O\left(N_{c}\right)$. This result depends on the instanton interaction, but as we noted above, $\gamma^{2}=O\left(1 / N_{c}\right)$ is a consequence of the fact that instantons are $S U(2)$ gauge field configurations.

There is a simple argument that explains why the instanton density scales as the number of colors. In our model, the size distribution is regularized by the interaction between instantons. This means that there has to be a balance between the average single instanton action and the average interaction between instantons. If the average instanton action satisfies $S_{0}=O\left(N_{c}\right)$ we expect that $\left\langle S_{\text {int }}^{\text {tot }}\right\rangle=O\left(N_{c}\right)$ also. Using $\left\langle S_{\text {int }}^{\text {tot }}\right\rangle=(N / V)\left\langle S_{\text {int }}\right\rangle$ and the fact that the average interaction between any two instantons satisfies $\left\langle S_{\text {int }}\right\rangle=O(1)$ we expect that the density grows as $N_{c}$.

Fig. 2 shows the instanton size distribution for different numbers of colors. We observe that the number of small instantons is strongly suppressed as $N_{c} \rightarrow \infty$ but the average size stabilizes at a finite value $\bar{\rho}<\Lambda^{-1}$. We also note that there is critical size $\rho^{*}$ for which the number of instantons does not change as $N_{c} \rightarrow \infty$. The value of $\rho^{*}$ is easy to determine analytically. We write $n(\rho)=\exp \left(N_{c} F(\rho)\right)$ with $F(\rho)=a \log (\rho)+b \rho^{2}+c$ where 
the coefficients $a, b, c$ are independent of $N_{c}$ in the large $N_{c}$ limit. The critical value of $\rho$ is given by the zero of $F(\rho)$. We find $\rho^{*}=0.49 \Lambda^{-1}$. The existence of a critical instanton size for which $n\left(\rho^{*}\right)$ is independent of $N_{c}$ was discussed by [24, 25, 26]. The problem was studied on the lattice by Lucini and Teper [27], who find $\rho^{*}=6 a=0.43 \mathrm{fm}$.

Next we wish to study fluctuations in the instanton liquid. Fluctuations in the net instanton number are related to the second derivative of the free energy with respect to $N$. We find

$$
\left\langle N^{2}\right\rangle-\langle N\rangle^{2}=\frac{4}{b}\langle N\rangle
$$

This result is in agreement with a low energy theorem based on broken scale invariance [28]

$$
\frac{1}{\left(32 \pi^{2}\right)^{2}} \int d^{4} x\left\{\left\langle g^{2} G^{2}(0) g^{2} G^{2}(x)\right\rangle-\left\langle g^{2} G^{2}(0)\right\rangle^{2}\right\}=\frac{4}{b} \frac{1}{32 \pi^{2}}\left\langle g^{2} G^{2}\right\rangle .
$$

This result is very general and based solely on the renormalization group equations. The left hand side is given by an integral over the field strength correlator, suitably regularized and with the constant term $\left\langle G^{2}\right\rangle^{2}$ subtracted. For a dilute system of instantons equ. (28) reduces to equ. (27). The result (27) shows that fluctuations of the instanton ensemble are suppressed by $1 / N_{c}$. This is agreement with general arguments showing that fluctuations are suppressed in the large $N_{c}$ limit. We also note that the result (27) clearly shows that even if instantons are semi-classical, interactions between instantons are crucial in the large $N_{c}$ limit.

Fluctuation in the topological charge can be studied by adding a $\theta$-term to the partition function (14). We find

$$
\left\langle Q^{2}\right\rangle=\langle N\rangle
$$

which is identical to the result in the random instanton liquid and not in agreement with Witten's hypothesis $\chi_{t o p}=O(1)$. However, Diakonov et al. noticed that equ. (29) is a consequence of the fact that in the sum ansatz the average interaction between instantons of the same charge is identical to the average interaction between instantons of opposite charge [29]. In general there is no reason for this to be the case and more sophisticated instanton interactions do not have this feature [30, 31, 32]. If $r$ denotes the ratio of the average interaction between instantons of opposite charge and instanton of the same charge, $r=\left\langle S_{I A}\right\rangle /\left\langle S_{I I}\right\rangle$, then 29]

$$
\left\langle Q^{2}\right\rangle=\frac{4}{b-r(b-4)}\langle N\rangle
$$


This result shows that for any value of $r \neq 1$ fluctuations in the topological charge are suppressed as $N_{c} \rightarrow \infty$. We also note that $\chi_{t o p}=O(1)$, in agreement with Witten's hypothesis.

\section{CHIRAL SYMMETRY BREAKING}

In this section we wish to study chiral symmetry breaking in the mean field approximation. This can be done by studying the Dyson-Schwinger equation for the quark propagator or by analyzing the spectrum of the Dirac operator. In this section we wish to use the more microscopic approach and analyze the spectrum of the Dirac operator. In a basis spanned by the individual zero modes of the instantons and anti-instantons the Dirac operator has the structure

$$
(i \not D)=\left(\begin{array}{cc}
0 & T_{I A} \\
T_{I A}^{\dagger} & 0
\end{array}\right),
$$

where $T_{I A}$ is the overlap matrix element of the Dirac operators between an instanton and anti-instanton zero mode. The matrix elements depend on the collective coordinates of the instanton. If the interaction between instantons is weak, the matrix elements are distributed randomly with zero average, but the second moment of $T_{I A}$ is non-zero. Averaging over the positions and orientations of the instantons we get

$$
\left\langle\left|T_{I A}^{2}\right|\right\rangle=\frac{2 \pi^{2}}{3 N_{c}} \frac{N \rho^{2}}{V} .
$$

The factor $1 / N_{c}$ comes from the average over $S U\left(N_{c}\right)$. Equ. (32) implies that the average matrix element of the Dirac operators decreases as $1 / N_{c}$ but the second moment in the zero modes zone is $O(1)$. If the matrix elements are distributed according to a Gaussian unitary ensemble, the spectral density is a semi-circle

$$
\rho(\lambda)=\frac{N}{\pi \sigma V}\left(1-\frac{\lambda^{2}}{4 \sigma^{2}}\right)^{1 / 2},
$$

with $\sigma^{2}=\left|T_{I A}^{2}\right|$. We observe that the width of the zero mode zone is related to $\sigma$, which is $O(1)$. According to the Banks-Casher formula the quark condensate is related to the spectral density at zero virtuality

$$
\langle\bar{q} q\rangle=-\frac{1}{\pi \rho}\left(\frac{3 N_{c}}{2} \frac{N}{V}\right)^{1 / 2} .
$$


Because $(N / V)=O\left(N_{c}\right)$ the quark condensate also grows as $N_{c}$. This, of course, is the expected behavior. We note, however, that the linear growth in $N_{c}$ is really a combination of two effects. The linear increase in the number of modes $N$ provides one factor of $N_{c}^{1 / 2}$ and the decrease in the average matrix element $\left|T_{I A}\right|$ contributes another factor of $N_{c}^{1 / 2}$.

The true spectral density of the Dirac operator in not given by a semi-circle. Schematically, the spectrum of the Dirac operator in quenched QCD is shown in Fig. B. There are several notable features. First, in a finite volume there is a certain number of exact zero modes. This number is proportional to $\left(\chi_{t o p} V\right)^{1 / 2}$ and therefore scales as $N_{c}^{0}$. We note that in the infinite volume limit exact zero modes are not important because their number scales as $\sqrt{V}$ while the total number of states increases linearly with the volume. The second feature of the spectrum is the logarithmic enhancement of the spectrum at small virtuality. This enhancement is an artifact of the quenched approximation. From quenched chiral perturbation theory we expect 33

$$
\rho(\lambda)=\frac{\Sigma}{\pi}\left\{1-\frac{m_{0}^{2}}{16 \pi^{2} f_{\pi}^{2}} \log \left(\frac{|\lambda|}{\mu}\right)+\ldots\right\} .
$$

Here, $\Sigma$ is a parameter that corresponds to the (negative) unquenched chiral condensate and $m_{0}$ is the mass of the quenched ghost pole. This mass corresponds to the mass of the $\eta^{\prime}$ in the chiral limit of full QCD. Using $m_{0}^{2}=O\left(1 / N_{c}\right)$ we find that the coefficient of the logarithmic enhancement vanishes as $1 / N_{c}$ in the large $N_{c}$ limit. This, of course, is consistent with the idea that the fermion determinant is not important in the large $N_{c}$ limit. The third component of the spectrum is given by the almost zero modes related to chiral symmetry breaking. This part of the spectrum is expected to scale as $N_{c}$, as is the bulk of the spectrum which is not related to chiral symmetry breaking.

At finite $N_{c}$ the spectrum of the Dirac operator in full QCD with light fermions has a different behavior. The number of exact zero modes is again proportional to $\left(\chi_{\text {top }} V\right)^{1 / 2}$ but in full QCD the topological susceptibility is suppressed, $\chi_{\text {top }} \simeq-m\langle\bar{\psi} \psi\rangle$. This implies that as $N_{c}$ increases the number of exact zero modes initially increases as $N_{c}^{1 / 2}$ and then saturates at the value corresponding to the quenched topological susceptibility. In the infinite volume limit the spectrum near the origin is linear. Chiral perturbation theory predicts the slope of the spectrum [34]

$$
\rho(\lambda)=\frac{\Sigma}{\pi}\left\{1+\frac{\left(N_{f}^{2}-4\right) \Sigma}{32 \pi N_{f} f_{\pi}^{4}}|\lambda|+\ldots\right\} .
$$


We observe that the constant part of the spectrum grows as $N_{c}$ whereas the linear part is independent of $N_{c}$. This implies that in the large $N_{c}$ limit the spectrum at the origin is flat for any number of flavors as long as $N_{f}$ is not of the order $N_{c}$.

\section{THE INTERACTING INSTANTON LIQUID}

In this section we wish to go beyond the mean field approximation and study the partition function of the instanton liquid using numerical simulations. These simulations take into account all correlations between instantons. The numerical techniques are described in detail in [35, 36]. In order to perform these simulations we have to fully specify the instanton interaction. We have used the "streamline" interaction determined in [31, 32]. The streamline solution is characterized by the fact that the action of the approximate instanton-anti-instanton solution is a local minimum except in the direction of the "valley" in configuration space that connects a well separated IA pair with a very close pair. There is no interaction between two instantons of the same charge. The interaction between two instantons of opposite charge approaches $-2 S_{0}$ if the relative color orientation is attractive.

This implies that the streamline interaction lacks the repulsive core that is required to stabilize the instanton ensemble at the classical level. In order to correct this problem we have added a purely phenomenological core to the instanton interaction. The interaction is given by

$$
S_{\text {core }}=\frac{8 \pi^{2}}{g^{2}} \frac{A}{\lambda^{4}}|u|^{2}, \quad \lambda=\frac{R^{2}+\rho_{I}^{2}+\rho_{A}^{2}}{2 \rho_{I} \rho_{A}}+\left(\frac{\left(R^{2}+\rho_{I}^{2}+\rho_{A}^{2}\right)^{2}}{4 \rho_{I}^{2} \rho_{A}^{2}}-1\right)^{1 / 2}
$$

in both $I I$ and $I A$ channels. The dimensionless parameter $A$ controls the strength of the core and is adjusted to reproduce the phenomenological diluteness $\rho^{4}(N / V)$ of the instanton ensemble. In our simulations we have used $A=128$. We note that the hard core interaction equ. (37) only acts between instantons that overlap in color space. As a result, we expect $\left\langle S_{\text {core }}\right\rangle=O(1)$, in agreement with the interaction used in the mean field treatment.

One might argue that there should not be any interaction between instantons of the same charge because there is a $2 \times\left(2 N_{c}\right)$ parameter family of two-instanton solutions with action $2 \times\left(8 \pi^{2}\right) / g^{2}$ [38]. However, there are two phenomena that lead to an effective instanton-

instanton interaction. The first is related to the fact that the collective coordinate measure for two close instantons is not just the product of two single instantons measures. Carter and 
Shuryak argued that this will lead to an effective short range instanton-instanton repulsion [39, 40]. Whether this effect has the same $N_{c}$ dependence as the classical interaction equ. (37) is an important problem. The second effect is that quantum corrections to a charge two instanton solution do not factorize. As a quantum correction, this effect is naively suppressed by $1 / S_{0} \sim 1 / N_{c}$, but the suppression can be overcome if instantons with different color orientation interact.

In order to determine the instanton density and the free energy of the instanton liquid we have to compute the partition function equ. (14). Monte Carlo simulations are ideally suited for computing expectation values, but they do not directly provide the partition function. It is possible, however, to compute free energy differences. This implies that Monte Carlo simulations can be used to compute the ratio of two partition functions. In practice we calculate the ratio of the exact partition function and the variational estimate equ. (19). The exact partition function is given by 36]

$$
\log Z=\log \left(Z_{1}\right)-\int_{0}^{1} d \alpha\left\langle\left(S-S_{1}\right)\right\rangle_{\alpha}
$$

Here, $Z_{1}$ is the variational partition function equ. (19) and $S_{1}$ is the variational action. The expectation value $\langle.\rangle_{\alpha}$ is determined using the interpolating action $S_{\alpha}=S_{1}+\alpha\left(S-S_{1}\right)$. $S_{\alpha}$ reduces to the variational action for $\alpha=0$ and the exact action for $\alpha=1$.

The free energy energy of the instanton liquid as a function of the instanton density for $N_{c}=3, \ldots, 6$ is shown in Fig. 4 . The equilibrium density is determined by the minimum of the function $F(N / V)$. The dependence of equilibrium density and the free energy on the number of colors is shown in Fig. 5. We observe that $(N / V)$ increases linearly with $N_{c}$ whereas the free energy is quadratic. The slope of $(N / V)$ as a function of $N_{c}$ is small, in agreement with the mean field result shown in Fig. 1. In contrast to the mean field result the linear behavior already sets in at small $N_{c} \simeq 3$. We have checked the stability of our results to changing the strength of the hard core interaction and including higher order corrections in the QCD beta function. Both changes affect the results quantitatively but not qualitatively. However, the results are crucially dependent on the assumption that the parameter $A$ in equ. (37) is not a function of $N_{c}$.

We have also studied the instanton size distribution, the topological susceptibility and the spectrum of the Dirac operator for different numbers of colors. In order to be able to distinguish more clearly between different scenarios we have not used the exact instanton 
density determined in Fig. 4 but have simply scaled $(N / V) \sim N_{c}$. At large $N_{c}$ this will only introduce errors that are suppressed by $1 / N_{c}$. The instanton size distribution is shown in Fig. 6. As expected small instantons are strongly suppressed as the number of colors increases. We observe a clear fixed point in the size distribution at $\rho^{*} \Lambda \simeq 0.27$.

Our simulations were carried out in the total topological charge $Q_{t o p}=0$ sector of the theory. We can nevertheless determine the topological susceptibility by measuring the average $Q_{\text {top }}^{2}$ in a sub-volume $V_{3} \times l_{4}$ of the euclidean box $V_{3} \times L_{4}$ 35. The finite volume susceptibility is given by

$$
\chi_{\text {top }}\left(l_{4}\right)=\frac{\left\langle Q_{\text {top }}^{2}\right\rangle_{V_{3} \times l_{4}}}{V_{3} \times l_{4}}\left(1-\frac{l_{4}}{L_{4}}\right)^{-1} .
$$

The factor $\left(1-l_{4} / L_{4}\right)^{-1}$ takes into account the constraint from overall charge neutrality. This correction factor is derived under the assumption that the fluctuations are Gaussian. In an ideal calculation $L_{4} \gg l_{4}$ and the correction for overall neutrality is small. The topological susceptibilities are shown in Fig. 7. We observe that $\chi_{\text {top }}\left(l_{4}\right)$ tends to a constant as $l_{4}$ increases. We identify this constant with the susceptibility in the thermodynamic limit. We find that for $N_{c}=3$ the topological susceptibility agrees well with the expectation based on Poissonian statistics, $\chi_{t o p} \simeq(N / V)$. For $N_{c}>3$, however, fluctuations are significantly suppressed and the topological susceptibility increases more slowly than the density of instantons. Fig. 8 shows that our results are consistent with a scenario in which $\chi_{\text {top }}$ remains finite as $N_{c} \rightarrow \infty$.

In Fig. 9 we show the spectrum of the Dirac operator for $N_{c}=3, \ldots, 6$. Since we calculate in the $Q_{t o p}=0$ sector of the theory there are no exact zero modes. We clearly observe the enhancement of the spectral density near $\lambda=0$, but we also note that this enhancement becomes weaker as the number of colors increases. The chiral condensate for $m_{q}=0.1 \Lambda$ is shown in Fig. 8. We clearly see that $\langle\bar{q} q\rangle$ is linear in $N_{c}$.

In the recent literature a number of authors have studied the role of instantons in chiral symmetry breaking by analyzing the local chirality $X(x)$ of low lying eigenstates of the Dirac operator [17]. The quantity $X(x)$ is defined by

$$
\tan \left(\frac{\pi}{4}(1+X(x))\right)=\left(\frac{\psi^{\dagger}\left(1+\gamma_{5}\right) \psi}{\psi^{\dagger}\left(1-\gamma_{5}\right) \psi}\right)^{1 / 2}
$$

where $\psi$ is an eigenfunction of the Dirac operator $i \not D \psi=\lambda \psi$ in a given gauge configuration. In order to study chiral symmetry breaking one only considers the lowest few eigenvec- 
tors. The instanton liquid model predicts that these eigenvectors are linear combinations of instanton and anti-instanton zero modes. This implies that at points $x$ where the wave function $\psi^{\dagger} \psi$ is large it is either left or right handed, $X(x) \simeq \pm 1$. In order to test this prediction one has to choose a cutoff on the eigenvalue $\lambda$ and some cutoff on the magnitude of $\psi^{\dagger} \psi$. Typically, the points $x$ are restricted to the top few percent of the eigenfunction. The instanton model suggests that this fraction should be no larger than the diluteness of the instanton liquid, and that the maximum eigenvalue should be smaller than the width $\left|T_{I A}\right|$ of the zero mode zone.

In Fig. 10 we show numerical results for the local chirality distribution in the instanton liquid model. We have included all states in the zero mode zone and used the top $30 \%$ of the wavefunction. The $N_{c}$ dependence is similar if more restrictive cuts are used. We observe that the double peak structure is very pronounced for all $N_{c}=3, \ldots, 6$. There is a small shift of the peaks toward smaller values of $X$ as the number of colors increases. We have verified that for $N_{c}>10$ the double peak structure disappears. This is related to the fact that the instanton density increases and instantons overlap in space. The instanton liquid remains dilute, however, because instantons do not overlap in color space. This is easily verified by computing chirality distributions for eigenstates of the Dirac operator projected on a $S U(2)$ subgroup. Cundy et al. computed local chirality distributions in lattice QCD for $N_{c}=2, \ldots, 5$ 41. In their calculations the double peak structure disappears much more quickly as the number of colors increases. This implies that either the instanton ensemble is not as dilute as we have assumed, or that mixing with non-zero modes is more important. Given the fact the value of $\rho^{*}$ in our simulations is also smaller than the value obtained by Lucini and Teper, it seems likely that the instanton liquid in QCD is not quite as dilute as suggested in [8].

\section{HADRONIC CORRELATION FUNCTIONS}

We have also studied hadronic correlation functions in the instanton liquid for different numbers of colors. We have considered, in particular, correlation functions of currents with the quantum numbers of the pion, the rho meson, and the $\eta^{\prime}$ meson. The currents are given by

$$
j_{\pi}=\bar{d} i \gamma_{5} u, \quad j_{\rho}^{\mu}=\bar{d} \gamma^{\mu} u, \quad j_{\eta^{\prime}}=\frac{1}{\sqrt{2}}\left(\bar{u} i \gamma_{5} u+\bar{d} i \gamma_{5} d\right)
$$


The pion and rho meson are flavor non-singlet mesons. The corresponding correlation functions only involve the connected contribution

$$
\Pi_{I=1}(x)=\left\langle\operatorname{Tr}\left[S^{a b}(0, x) \Gamma S^{b a}(x, 0) \Gamma\right]\right\rangle,
$$

with $\Gamma=i \gamma_{5}, \gamma_{\mu}$ for the pion and rho meson, respectively. Here, $S^{a b}$ denotes the quark propagator in a given gauge configuration, $a, b$ are color indices, and the trace runs over Dirac indices. The average $\langle$.$\rangle is performed with respect to the partition function equ. (14). The \eta^{\prime}$ meson is a flavor singlet meson and the correlation function has an additional, disconnected, term. We find

$$
\Pi_{I=0}(x)=\left\langle\operatorname{Tr}\left[S^{a b}(0, x) \Gamma S^{b a}(x, 0) \Gamma\right]\right\rangle-2\left\langle\operatorname{Tr}\left[S^{a a}(0,0) \Gamma\right] \operatorname{Tr}\left[S^{b b}(x, x) \Gamma\right]\right\rangle,
$$

with $\Gamma=i \gamma_{5}$. The masses of the $\pi, \rho$ and $\eta^{\prime}$ mesons can be extracted by fitting the correlation functions to a spectral representation. At large euclidean separation the correlation function is determined by the lowest hadronic resonance. A good model for the contribution of excited states is given by the free quark-anti-quark continuum above some threshold invariant mass $\sqrt{s_{0}}$. The threshold roughly corresponds to the location of the first excited state. For the pion and rho meson we use

$$
\begin{aligned}
& \Pi_{\pi}(x)=\lambda_{\pi}^{2} D\left(m_{\pi}, x\right)+\frac{N_{c}}{8 \pi^{2}} \int_{s_{0}}^{\infty} d s s D(\sqrt{s}, x), \\
& \Pi_{\rho}(x)=f_{\rho}^{2} m_{\rho}^{4} D\left(m_{\rho}, x\right)+\frac{2 N_{c}}{8 \pi^{2}} \int_{s_{0}}^{\infty} d s s D(\sqrt{s}, x),
\end{aligned}
$$

where $D(m, x)$ is the euclidean space propagator for a free scalar meson with mass $m$

$$
D(m, x)=\frac{m}{4 \pi^{2} x} K_{1}(m x) .
$$

In full QCD the $\eta^{\prime}$ meson can be described by the same spectral representation as the pion. In quenched $\mathrm{QCD}$, however, the spectral function in the $\eta^{\prime}$ channel is not positive definite. There is a well established method for dealing with this problem. In quenched chiral perturbation theory it is assumed that the disconnected part of the $\eta^{\prime}$ correlation function corresponds to a ghost pole in the spectrum [42, 43, 44]. In momentum space the pole contributions are given by

$$
\Pi_{\eta^{\prime}}(q)=\frac{\lambda_{\pi}^{2}}{q^{2}+m_{\pi}^{2}}-\frac{\lambda_{\pi}^{2}}{q^{2}+m_{\pi}^{2}} m_{0}^{2} \frac{1}{q^{2}+m_{\pi}^{2}} .
$$


Going from quenched to unquenched QCD corresponds to summing the geometric series

$$
\Pi_{\eta^{\prime}}(q) \rightarrow \frac{\lambda_{\pi}^{2}}{q^{2}+m_{\pi}^{2}+m_{0}^{2}}
$$

and $m_{\eta^{\prime}}^{2}=m_{0}^{2}+m_{\pi}^{2}$ in full QCD. This result is expected to be exact in the large $N_{c}$ limit. In addition to that there is evidence from the lattice that the mass of the $\eta^{\prime}$ meson can be extracted from the ghost pole propagator even for $N_{c}=3$ [44]. In coordinate space equ. (47) corresponds to

$$
\Pi_{\pi}(x)=\lambda_{\pi}^{2} D\left(m_{\pi}, x\right)-\lambda_{\pi}^{2} m_{0}^{2} D^{\prime}\left(m_{\pi}, x\right)+\frac{N_{c}}{8 \pi^{2}} \int_{s_{0}}^{\infty} d s s D(\sqrt{s}, x)
$$

with

$$
D^{\prime}(m, x)=\frac{1}{8 \pi^{2}} K_{0}(m x)
$$

We have used the spectral representation equ. (49) to determine the quenched $\eta^{\prime}$ mass $m_{0}$ for different $N_{c}$.

Before we come to the numerical studies we would like to present several analytical results. If we study correlation functions at distances small compared to the average separation between instantons, $x \ll(N / V)^{-1 / 4}$, it is sufficient to take into account the contribution from the closest instanton only. The quark propagator is given by

$$
S(x, y) \simeq \frac{\psi_{0}(x-z) \psi_{0}^{\dagger}(y-z)}{m^{*}}
$$

where $\psi_{0}(x)$ is the zero mode wave function and $z$ is the location of the instanton. The effect of all other instantons only enters via the effective mass $m^{*} \simeq \pi \rho\left(2 / N_{c}\right)^{1 / 2}(N / V)^{1 / 2}$. The single instanton contribution to the correlation function is found by inserting the propagator equ. (51) into the equations (42) and (43) and averaging over the collective coordinates of the instanton.

Because of the chiral structure of the rho meson current there is no zero mode contribution to the rho meson correlation function. In the pion and $\eta^{\prime}$ meson channel we find 45

$$
\Pi_{\pi, \eta^{\prime}}^{S I A}(x)= \pm \int d \rho n(\rho) \frac{6 \rho^{4}}{\pi^{2}} \frac{1}{\left(m^{*}\right)^{2}} \frac{\partial^{2}}{\partial\left(x^{2}\right)^{2}}\left\{\frac{4 \xi^{2}}{x^{4}}\left(\frac{\xi^{2}}{1-\xi^{2}}+\frac{\xi}{2} \log \frac{1+\xi}{1-\xi}\right)\right\}
$$

with $\xi^{2}=x^{2} /\left(x^{2}+4 \rho^{2}\right)$. As is well known, the instanton contribution is attractive in the pion channel, and repulsive in the $\eta^{\prime}$ channel. The contribution of one instanton of given size does not involve any factors of $N_{c}$, and is the same, up to the sign, in the pion and 
$\eta^{\prime}$ channel. This is illustrated in Fig. 11. Perturbative contributions to the disconnected correlation function are suppressed by a factor $1 / N_{c}$ compared to the connected correlator. However, the single instanton contribution to the disconnected correlator is exactly the same as the instanton contribution to the connected correlator. After integration over the instanton distribution the correlation function scales as $N_{c}$ because the instanton density is proportional to $N_{c}$. This is the expected behavior in the case of the pion correlation function but it implies that in the single instanton approximation the anomalous $\pi-\eta^{\prime}$ splitting does not disappear in the large $N_{c}$ limit.

In order to go to large distances, $x>(N / V)^{-1 / 4}$ we have to resum the instanton interaction. This can be achieved using the mean field (Hartree) and RPA methods [46]. The mean field approximation gives the constituent quark propagator

$$
S_{Q}(x)=\int \frac{d^{4} p}{(2 \pi)^{4}} e^{i p \cdot x} \frac{\not p+i M(p)}{p^{2}+M(p)^{2}},
$$

where $M(p)$ is the dynamically generated quark mass. The momentum dependence of $M$ is determined by the Fourier transform of zero mode wave function. In the rho meson channel there is no direct instanton induced interaction and the correlation function is given by two non-interacting constituent quarks. We have

$$
\Pi_{\Gamma}(x)^{M F A}=N_{c} \operatorname{Tr}\left[S_{Q}(x) \Gamma S_{Q}(-x) \Gamma\right]
$$

where the trace is over the Dirac indices only and $\Gamma=\gamma_{\mu}$. In the pion and $\eta^{\prime}$ channel there is a direct instanton interaction that can be resummed using the RPA. We have 10, 46, 47, 48

$$
\Pi_{\pi, \eta^{\prime}}(x)=\Pi_{\pi}^{M F A}(x)+\Pi_{\pi, \eta^{\prime}}^{R P A}
$$

with

$$
\Pi_{\pi, \eta^{\prime}}^{R P A}(x)=N_{c}\left(\frac{N_{c} V}{N}\right) \int d^{4} q e^{i q \cdot x} \Gamma_{5}(q) \frac{ \pm 1}{1 \mp C_{5}(q)} \Gamma_{5}(q) .
$$

The loop and vertex functions $C_{5}$ and $\Gamma_{5}$ are given by

$$
\begin{aligned}
C_{5}(q) & =4 N_{c}\left(\frac{V}{N}\right) \int \frac{d^{4} p}{(2 \pi)^{4}} \frac{M_{1} M_{2}\left(M_{1} M_{2}-p_{1} \cdot p_{2}\right)}{\left(M_{1}^{2}+p_{1}^{2}\right)\left(M_{2}^{2}+p_{2}^{2}\right)}, \\
\Gamma_{5}(q) & =4 \int \frac{d^{4} p}{(2 \pi)^{4}} \frac{\left(M_{1} M_{2}\right)^{1 / 2}\left(M_{1} M_{2}-p_{1} \cdot p_{2}\right)}{\left(M_{1}^{2}+p_{1}^{2}\right)\left(M_{2}^{2}+p_{2}^{2}\right)}
\end{aligned}
$$

where $p_{1}=p+q / 2, p_{2}=p-q / 2$ and $M_{1,2}=M\left(p_{1,2}\right)$. Using $(N / V) \sim N_{c}$ and $M \sim 1$ we observe that the pion and $\eta^{\prime}$ correlation functions in the large $N_{c}$ limit depend on $N_{c}$ only through an overall factor of $N_{c}$. This implies $f_{\pi}^{2}=O\left(N_{c}\right)$ and $m_{\pi}, m_{\eta^{\prime}}=O(1)$. 
Numerical results for the constituent quark mass $M_{Q}$ as well as the pion mass $m_{\pi}$ and pion decay constant $f_{\pi}$ are shown in Fig. 12. We have used the average instanton size and density determined in section III]. The current quark mass is $m_{q}=0.025 \Lambda$. There is some variation in the constituent quark and pion masses for small $N_{c}<10$ but the size of $1 / N_{c}$ corrections is not large, about $20 \%$ for $N_{c}=3$. The pion decay constant shows the expected $N_{c}^{1 / 2}$ behavior but in this case $1 / N_{c}$ corrections are large, about $80 \%$ for $N_{c}=3$.

In Fig. 13 we show the correlation functions in the pion, rho meson, and $\eta^{\prime}$ meson channel. The correlation functions are normalized to free field behavior. The overall factor of $N_{c}$ drops out if the correlator is normalized in this way. We observe that the $\rho$ meson correlation function is essentially independent of $N_{c}$ already for small $N_{c}$. There are substantial $1 / N_{c}$ corrections in the $\pi$ and $\eta^{\prime}$ channel. The splitting between the $\pi$ and $\eta^{\prime}$ correlation functions is reduced in going from $N_{c}=3$ to $N_{c}=6$ but it remains finite and large as $N_{c} \rightarrow \infty$.

The correlation functions measured in numerical simulations of the instanton liquid for $N_{c}=3, \ldots 6$ are shown in Fig. 14. The meson masses extracted from the spectral representation equs. (44,45,49) are shown in Fig. 15. The results were obtained from simulations with $N=128$ instantons in a euclidean volume $V \Lambda^{4}=V_{3} \times 5.76$. $V_{3}$ was adjusted such that $(N / V)=\left(N_{c} / 3\right) \Lambda^{4}$. In order to avoid finite volume artifacts the current quark mass was taken to be rather large, $m_{q}=0.2 \Lambda$. We observe that the rho meson correlation function exhibits almost perfect scaling with $N_{c}$ and as a result the rho meson mass is practically independent of $N_{c}$. The scaling is not as good in the case of the pion. As a consequence there is some variation in the pion mass. However, as one can see from the fit shown in Fig. 15, this effect is consistent with $1 / N_{c}$ corrections that amount to about $40 \%$ of the pion mass for $N_{c}=3$. Finally, we study the behavior of the $\eta^{\prime}$ correlation function. There is a clear tendency toward $U(1)_{A}$ restoration, but the correlation function is still very repulsive for $N_{c}=6$. As one can see from Fig. 15 the result is consistent with $m_{\eta^{\prime}}^{2} \sim 1 / N_{c}$ although the error bars are quite large.

For comparison, we show the expected behavior of the correlation functions based on standard large $N_{c}$ counting in Fig. 16. We have used the spectral representation equs. (44,49) together with the phenomenological values $m_{\pi}=139 \mathrm{MeV}, \lambda_{\pi}=(450 \mathrm{MeV})^{2}, E_{0}=1.3 \mathrm{GeV}$ and $m_{0}=900 \mathrm{MeV}\left(N_{c}=3\right)$. We assume that $\lambda_{\pi}^{2} \sim N_{c}$ and $m_{0}^{2} \sim 1 / N_{c}$. We observe that the $\eta^{\prime}$ correlation function only approaches the pion correlation for fairly large values of $N_{c}$. For example, the $\eta^{\prime}$ correlation function does not show intermediate range attraction unless 
$N_{c}>15$. The variation in going from $N_{c}=3$ to $N_{c}=6$ is not dramatic, in agreement with the results shown in Fig. 14.

\section{SUMMARY}

In summary we have studied instantons in the large $N_{c}$ limit of QCD. We have argued that it is possible for the instanton liquid model to have a smooth large $N_{c}$ limit which is in agreement with scaling relations derived from Feynman diagrams. In this limit the density of instantons grows as $N_{c}$ whereas the typical instanton size remains finite. Interactions between instanton are important and suppress fluctuations of the topological charge. As a result the $U(1)_{A}$ anomaly is effectively restored even though the number of instantons increases. Using mean field arguments [9] and numerical simulations we have shown that this scenario does not require fine tuning. It arises naturally if the instanton ensemble is stabilized by a classical repulsive core. In this case we obtain a picture in which the instanton density is large but the instanton liquid remains dilute because instantons are not strongly overlapping in color space. Further investigations will have to show whether this scenario is indeed correct. For example, it would be useful to study the exact moduli space for multi-instanton configurations in the large $N_{c}$ limit.

Of course, there is no a priori reason why instantons have to be compatible with standard large $N_{c}$ counting rules. Instantons are not part of the diagrammatic expansion and do not need to satisfy scaling relations derived from diagrams. On the other hand it would be hard to reconcile the quantitative success of the instanton liquid model in describing chiral symmetry breaking and the mass of the $\eta^{\prime}$ with the phenomenological success of the $1 / N_{c}$ expansion if instanton effects strongly violate $N_{c}$ counting rules.

We should emphasize that the numerical results presented in this work only cover fairly small values of $N_{c}, N_{c} \leq 6$, and that all the analytical results were obtained in the mean field approximation. We cannot exclude the possibility that there is a phase transition as the number of colors becomes large [24, 39, 49]. Carter and Shuryak suggested, for example, that for clusters involving $O\left(N_{c}\right)$ instantons $1 / N_{c}$ suppressed color singlet exchanges become dominant and lead to the formation of tightly bound molecules. We did not observe this phenomenon in our simulations even if a short range color singlet interaction was included, but the number of colors $\left(N_{c} \leq 10\right)$ may have been too small. We also did not investigate 
the possibility that instantons in the large $N_{c}$ limit melt or dissociate into constituents with fractional topological charge. The latter scenario was investigated in the case of the $C P^{N-1}$ model in [50].

Our results can be compared to the lattice results of Lucini and Teper [27] and Cundy, Teper and Wenger [41]. Lucini and Teper find a fixed point in the instanton size distribution, in agreement with our results shown in Figs. 20 and 6. However, they do not find any suppression of large size instantons. Cundy et al. studied the local chirality distribution. They find that the double peak structure of this distribution disappears in the large $N_{c}$ limit. Our results shown in Fig. 10 are in qualitative, but not in quantitative agreement with their findings. In our calculations the effect is much less pronounced. This implies that either the instanton liquid is not as dilute as it is in our calculations, that mixing with non-zero modes becomes more important as $N_{c}$ increases, or that instantons are no longer semi-classical.

Acknowledgments: I would like to thank D. Diakonov, D. Gross, E. Shuryak, U. Wenger and A. Zhitnitsky for useful discussions. I would also like to acknowledge the hospitality of the Institute for theoretical Physics at UCSB where this work was completed. This work was supported in part by US DOE grant DE-FG-88ER40388 and by the National Science Foundations under grant No. PHY99-07949. 
[1] G. 't Hooft, Nucl. Phys. B 72, 461 (1974).

[2] E. Witten, Nucl. Phys. B 149, 285 (1979).

[3] E. Witten, Phys. Rev. Lett. 81, 2862 (1998) hep-th/9807109.

[4] E. Witten, Nucl. Phys. B 156, 269 (1979).

[5] G. Veneziano, Nucl. Phys. B 159, 213 (1979).

[6] A. A. Belavin, A. M. Polyakov, A. S. Shvarts and Y. S. Tyupkin, Phys. Lett. B 59, 85 (1975).

[7] C. G. Callan, R. F. Dashen and D. J. Gross, Phys. Rev. D 17, 2717 (1978).

[8] E. V. Shuryak, Nucl. Phys. B 203, 93 (1982).

[9] D. Diakonov and V. Y. Petrov, Nucl. Phys. B 245, 259 (1984).

[10] T. Schäfer and E. V. Shuryak, Rev. Mod. Phys. 70, 323 (1998) hep-ph/9610451.

[11] E. V. Shuryak, Rev. Mod. Phys. 65, 1 (1993).

[12] T. Schäfer and E. V. Shuryak, Phys. Rev. Lett. 86, 3973 (2001) hep-ph/0010116.

[13] M. Teper, Nucl. Phys. Proc. Suppl. 83, 146 (2000) hep-lat/9909124.

[14] M. C. Chu, G. M. Grandy, S. Huang, and J. W. Negele, Phys. Rev. D 496039 (1994). hep-lat/9312071.

[15] D. Diakonov and V. Y. Petrov, Sov. Phys. JETP 62204 (1985).

[16] T. DeGrand and A. Hasenfratz, Phys. Rev. D 64, 034512 (2001) hep-lat/0012021.

[17] I. Horvath, N. Isgur, J. McCune and H. B. Thacker, Phys. Rev. D 65, 014502 (2002) hep-lat/0102003]. T. DeGrand and A. Hasenfratz, Phys. Rev. D 65, 014503 (2002) [heplat/0103002 . I. Hip, T. Lippert, H. Neff, K. Schilling and W. Schroers, Phys. Rev. D 65, 014506 (2002) hep-lat/0105001. R. G. Edwards and U. M. Heller, Phys. Rev. D 65, 014505 (2002) hep-lat/0105004]. T. Blum et al., Phys. Rev. D 65, 014504 (2002) hep-lat/0105006. C. Gattringer, M. Gockeler, P. E. Rakow, S. Schaefer and A. Schafer, Nucl. Phys. B 617, 101 (2001) hep-lat/0107016. S. J. Dong et al., Nucl. Phys. Proc. Suppl. 106, 563 (2002) hep-lat/0110037.

[18] G. 't Hooft, Phys. Rev. Lett. 37, 8 (1976).

[19] M. A. Shifman, A. I. Vainshtein and V. I. Zakharov, Nucl. Phys. B 163, 46 (1980).

[20] E. M. Ilgenfritz and M. Muller-Preussker, Nucl. Phys. B 184, 443 (1981).

[21] G. Munster and C. Kamp, Eur. Phys. J. C 17, 447 (2000) hep-th/0005084. 
[22] G. 't Hooft, Phys. Rev. D 14, 3432 (1976) [Erratum-ibid. D 18, 2199 (1978)].

[23] This behavior was first observed in E. M. Ilgenfritz and M. Muller-Preussker, Phys. Lett. B 99, 128 (1981). These authors find $(N / V)=O(1)$ rather than $(N / V)=O\left(N_{c}\right)$ because they assume that there is a hard core interaction even between instantons that do not overlap in color space.

[24] H. Neuberger, Phys. Lett. B 94, 199 (1980).

[25] M. J. Teper, Z. Phys. C 5, 233 (1980).

[26] E. V. Shuryak, Phys. Rev. D 52, 5370 (1995) hep-ph/9503467.

[27] B. Lucini and M. Teper, JHEP 0106, 050 (2001) hep-lat/0103027.

[28] V. A. Novikov, M. A. Shifman, A. I. Vainshtein and V. I. Zakharov, Nucl. Phys. B 191, 301 (1981).

[29] D. Diakonov, M. V. Polyakov and C. Weiss, Nucl. Phys. B 461, 539 (1996) hep-ph/9510232].

[30] E. V. Shuryak, Nucl. Phys. B 319, 521 (1989).

[31] V. V. Khoze and A. Ringwald, Phys. Lett. B 259, 106 (1991).

[32] J. J. Verbaarschot, Nucl. Phys. B 362, 33 (1991) [Erratum-ibid. B 386, 236 (1992)].

[33] D. Toublan and J. J. Verbaarschot, Nucl. Phys. B 560, 259 (1999) hep-th/9904199.

[34] A. V. Smilga and J. Stern, Phys. Lett. B 318, 531 (1993).

[35] E. V. Shuryak and J. J. Verbaarschot, Phys. Rev. D 52, 295 (1995) hep-lat/9409020.

[36] T. Schäfer and E. V. Shuryak, Phys. Rev. D 53, 6522 (1996) hep-ph/9509337.

[37] T. Schäfer and E. V. Shuryak, Phys. Rev. D54, 1099 (1996) hep-ph/9512384.

[38] M. F. Atiyah, N. J. Hitchin, V. G. Drinfeld and Y. I. Manin, Phys. Lett. A 65, 185 (1978).

[39] G. W. Carter and E. V. Shuryak, Phys. Lett. B 524, 297 (2002).

[40] M. Garcia Perez, T. G. Kovacs and P. van Baal, Phys. Lett. B 472, 295 (2000) [hep$\mathrm{ph} / 9911485$.

[41] N. Cundy, M. Teper and U. Wenger, hep-lat/0203030.

[42] S. R. Sharpe, Phys. Rev. D 46, 3146 (1992) hep-lat/9205020].

[43] C. W. Bernard and M. F. Golterman, Phys. Rev. D 46, 853 (1992) hep-lat/9204007.

[44] Y. Kuramashi, M. Fukugita, H. Mino, M. Okawa and A. Ukawa, Phys. Rev. Lett. 72, 3448 (1994).

[45] E. V. Shuryak, Nucl. Phys. B 214, 237 (1983).

[46] D. Diakonov and V. Y. Petrov, Nucl. Phys. B 272, 457 (1986). 
[47] M. Kacir, M. Prakash and I. Zahed, Acta Phys. Polon. B 30, 287 (1999) hep-ph/9602314.

[48] M. Hutter, Z. Phys. C 74, 131 (1997) hep-ph/9501245.

[49] D. J. Gross and A. Matytsin, Nucl. Phys. B 429, 50 (1994) hep-th/9404004.

[50] D. Diakonov and M. Maul, Nucl. Phys. B 571, 91 (2000) hep-th/9909078. 


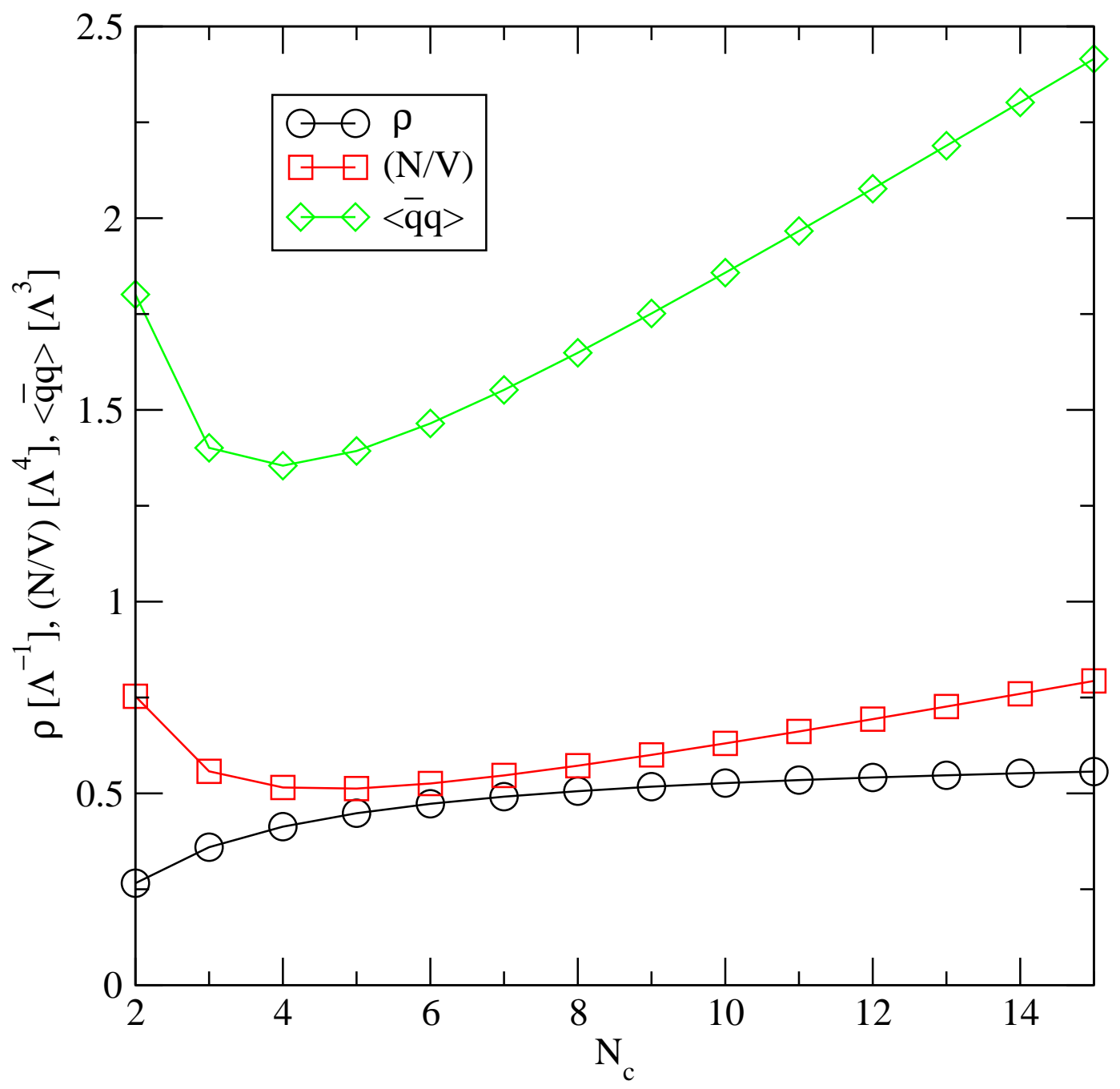

FIG. 1: Average instanton size $\rho$, instanton density $(N / V)$ and quark condensate $\langle\bar{q} q\rangle$ for different numbers of colors $N_{c}$. The results shown in this figure were obtained using the mean field approximation. All quantities are given in units of the QCD scale parameter $\Lambda$. 


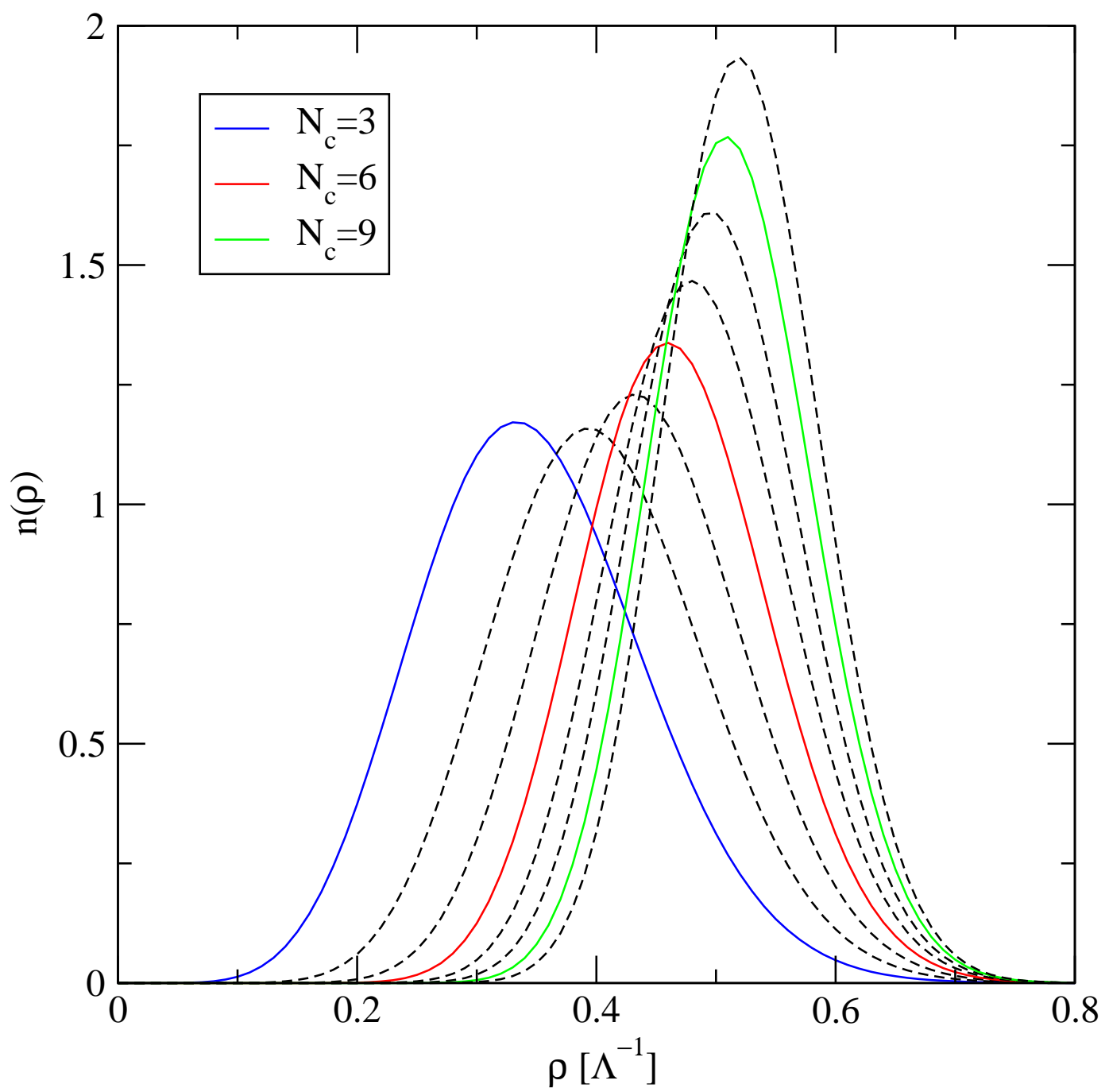

FIG. 2: Instanton size distribution $n(\rho)$ for different numbers of colors $N_{c}=3, \ldots, 10$. The results show in this figure were obtained using the mean field approximation. 


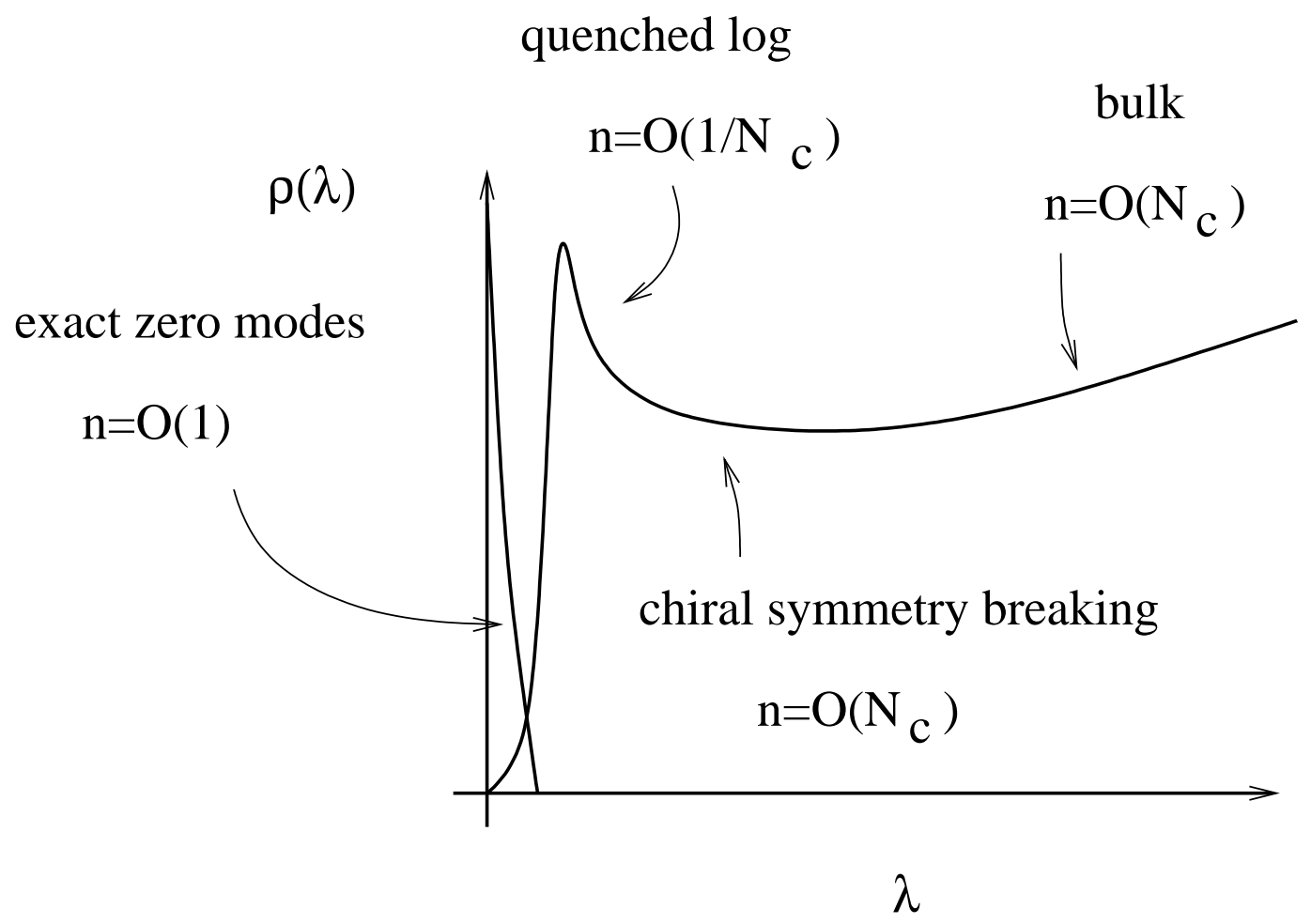

FIG. 3: Schematic behavior of the spectrum of the Dirac operator in quenched QCD with many colors. 


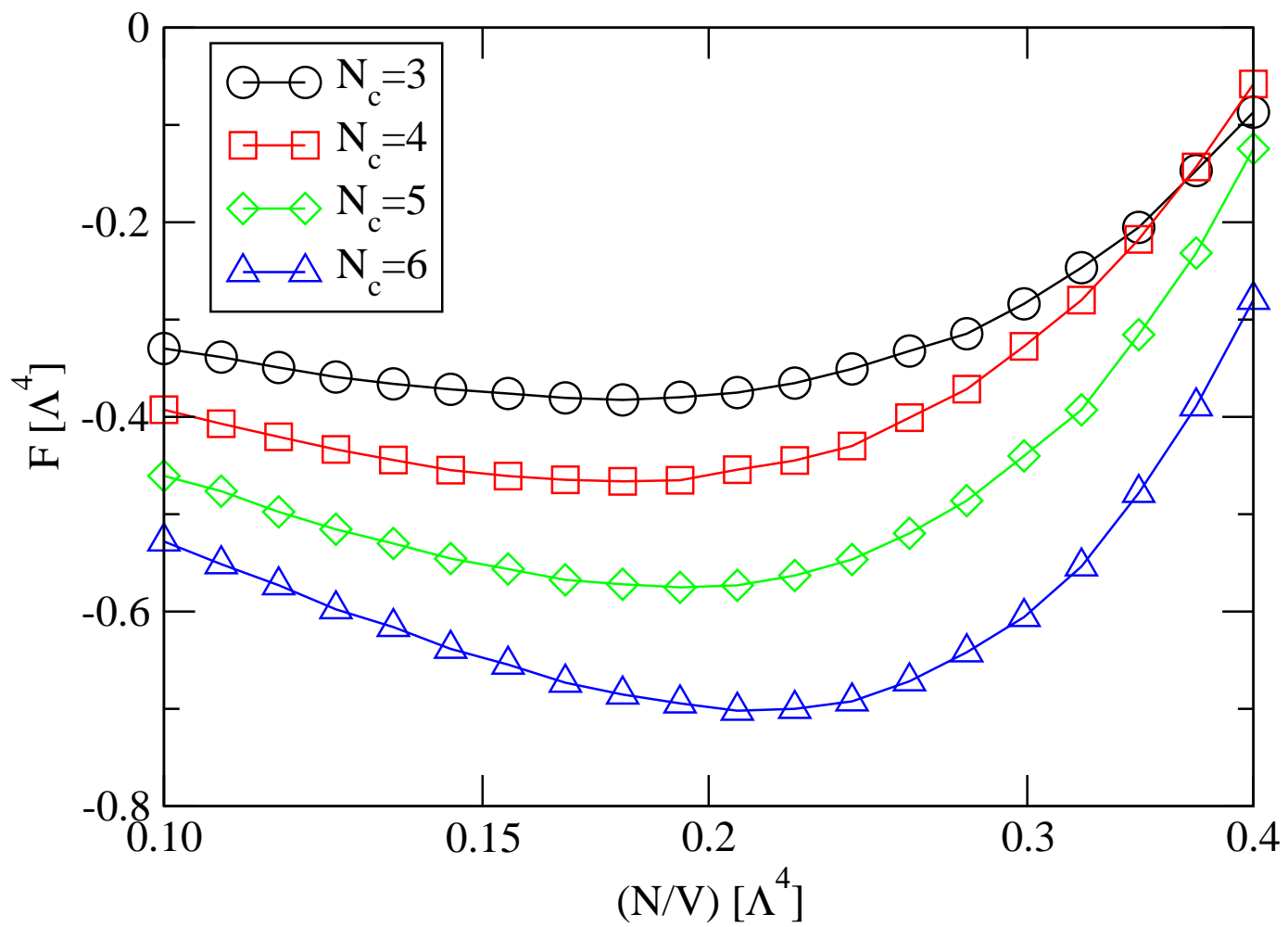

FIG. 4: Free energy $F$ of the quenched instanton liquid as a function of the instanton density $(N / V)$ for $N_{c}=3, \ldots, 6$ colors. Both $(N / V)$ and $F$ are given in units of the QCD scale parameter. The results shown in this figure were obtained using numerical simulations with $N=32$ instantons. 


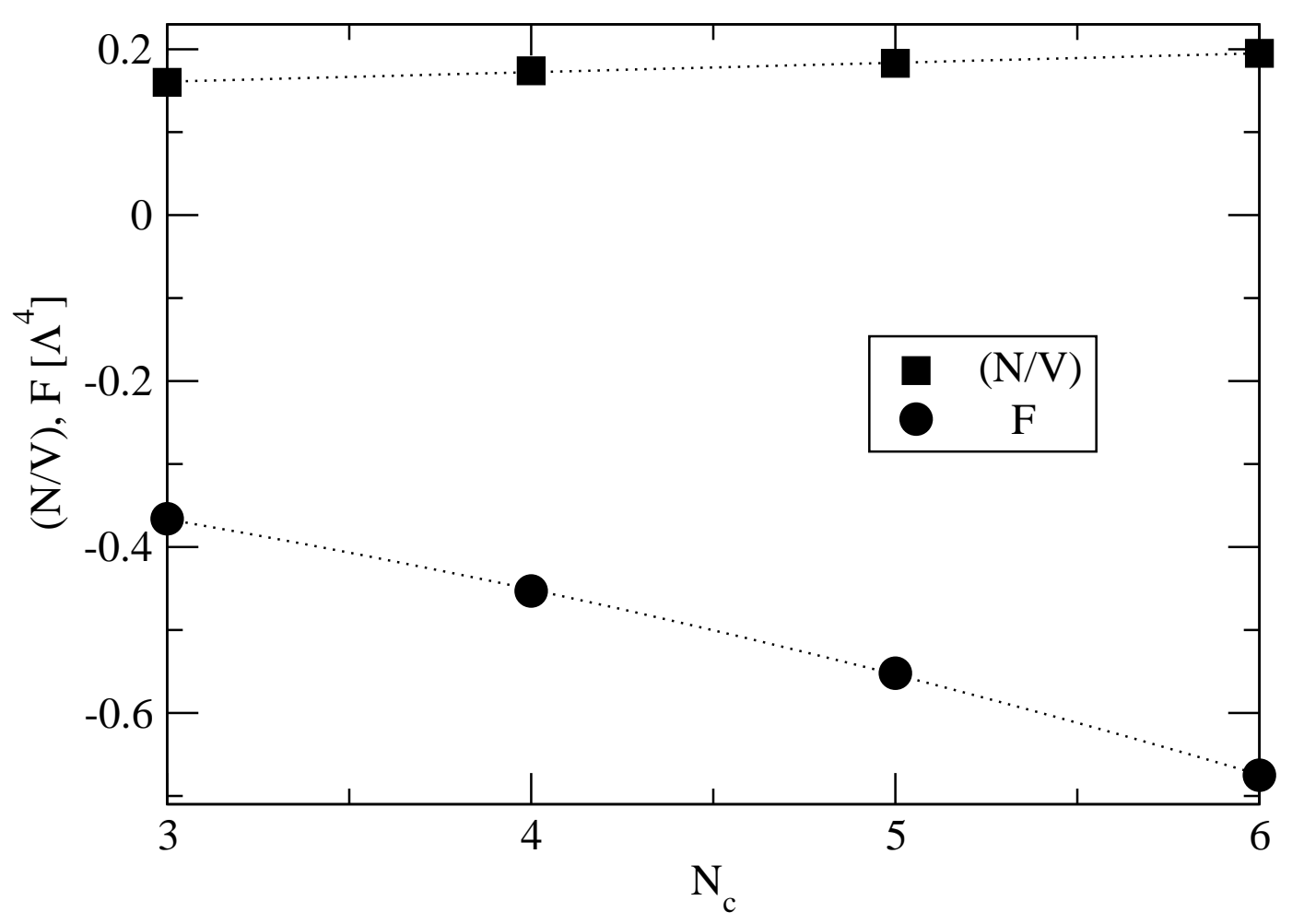

FIG. 5: Instanton density $(N / V)$ and free energy $F$ in a pure gauge instanton ensemble for $N_{c}=$ $3, \ldots, 6$ colors. Both $(N / V)$ and $F$ are given in units of $\Lambda^{4}$ where $\Lambda$ is the QCD scale parameter. The dashed lines show fits of the form $a_{1} N_{c}^{2}+a_{2} N_{c}+a_{3}$ (for the free energy $F$ ) and $a_{2} N_{c}+a_{3}$ (for the instanton density $N / V)$. 


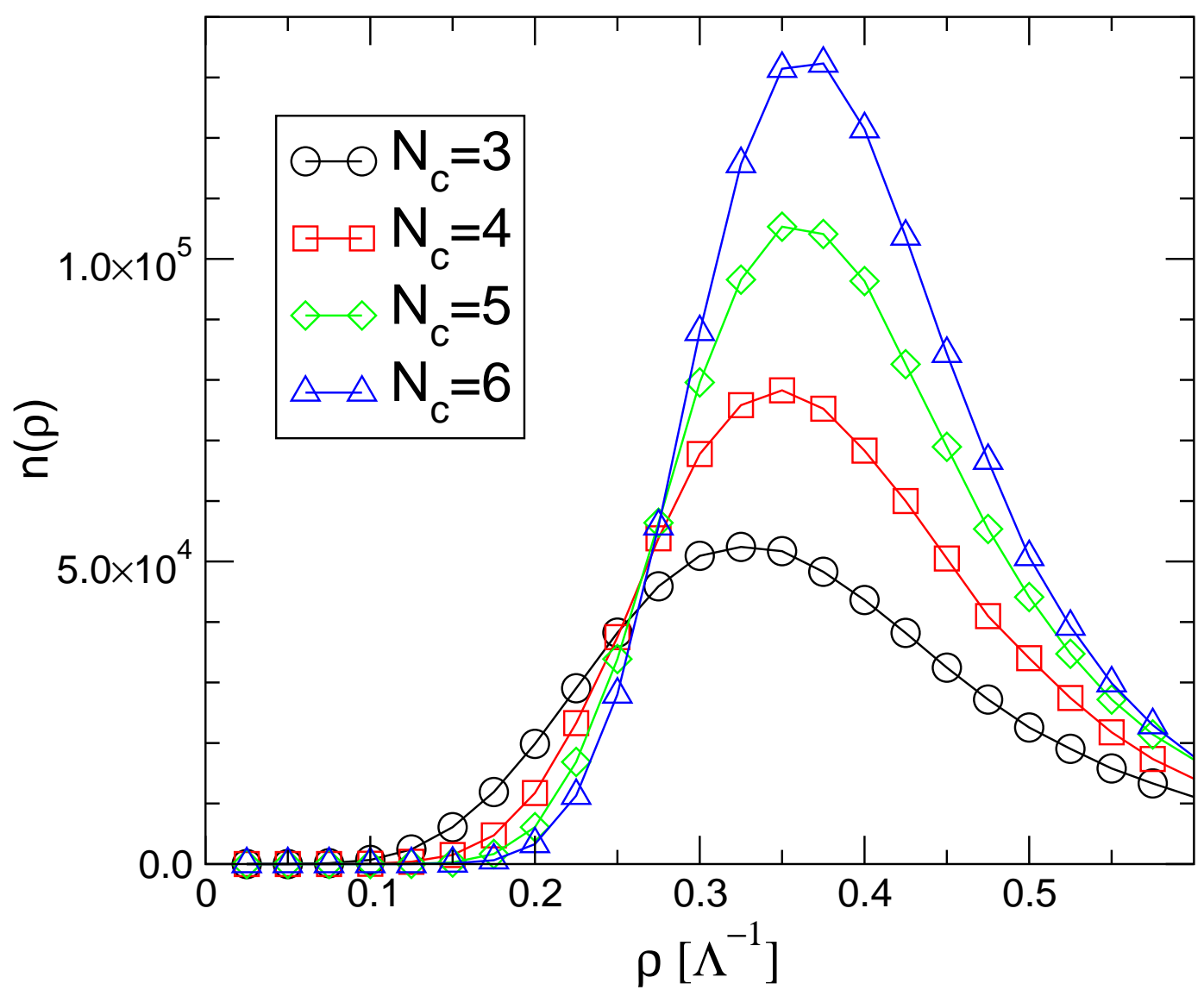

FIG. 6: Instanton size distribution in a pure gauge instanton ensemble for different numbers of colors. The results were obtained using numerical simulations with $N=128$ instantons. 


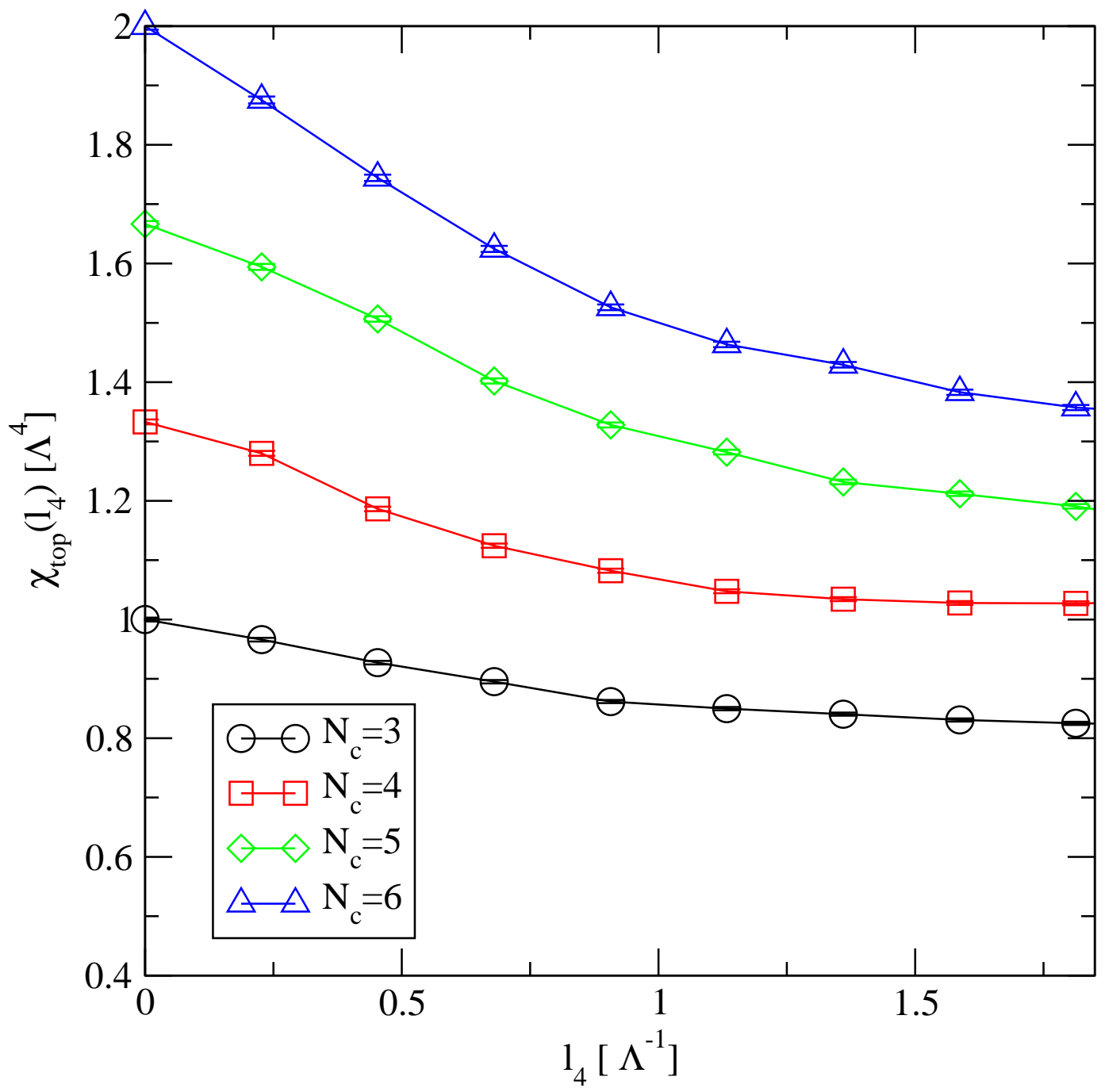

FIG. 7: Finite volume topological susceptibility $\chi_{t o p}\left(l_{4}\right)$ in a pure gauge instanton ensemble for different numbers of colors. The results were obtained using numerical simulations with $N=128$ instantons. 


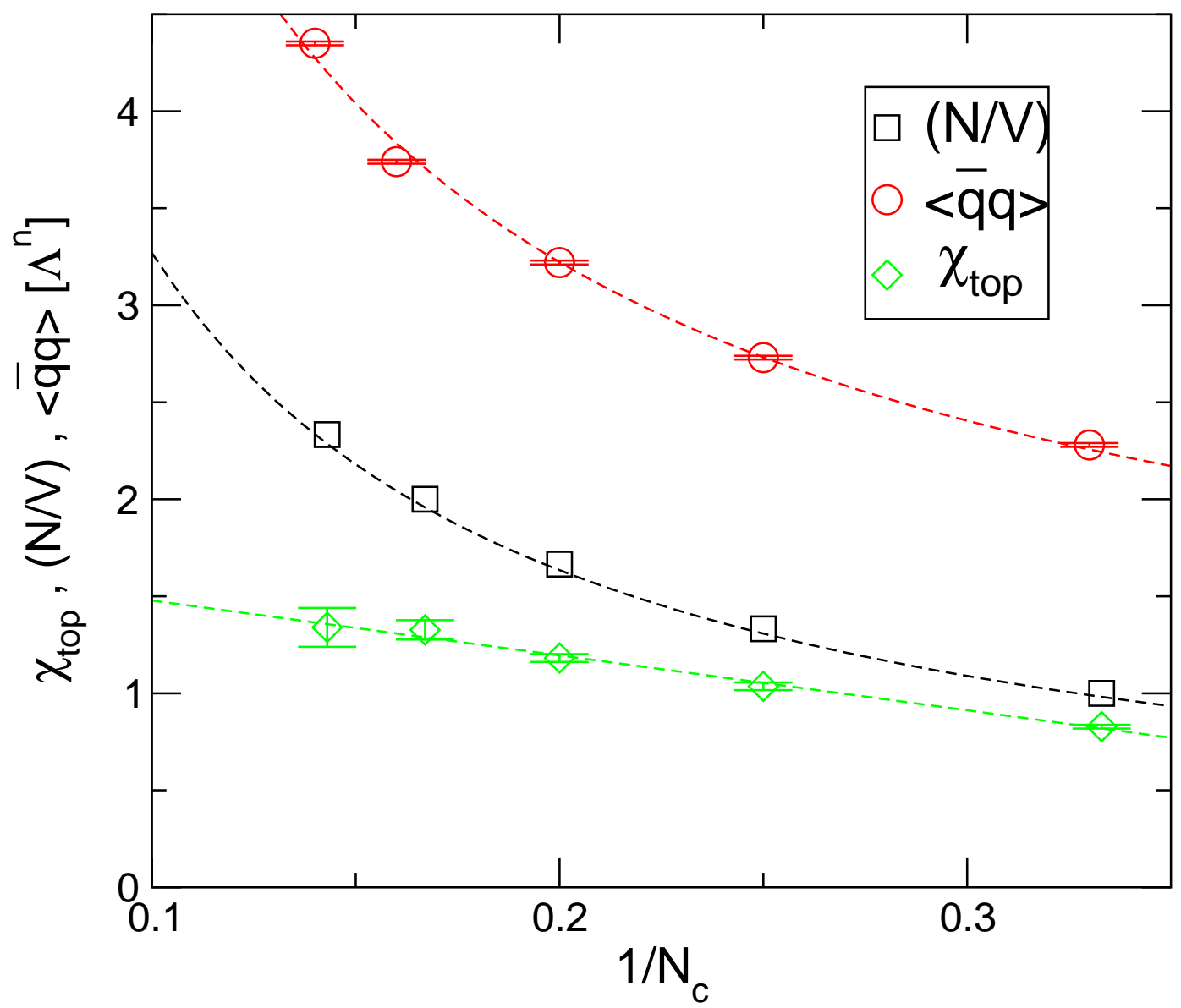

FIG. 8: Dependence of the chiral condensate $\langle\bar{\psi} \psi\rangle$ and the topological susceptibility $\chi_{\text {top }}$ on the number of colors. The instanton density $(N / V)$ was assumed to scale as $(N / V) \sim N_{c}$. The dashed lines show fits of the form $a_{1} N_{c}+a_{2}$ (for $\langle\bar{\psi} \psi\rangle$ and $\left.N / V\right)$ and $a_{2}+a_{3} / N_{c}$ (for $\chi_{t o p}$ ). 


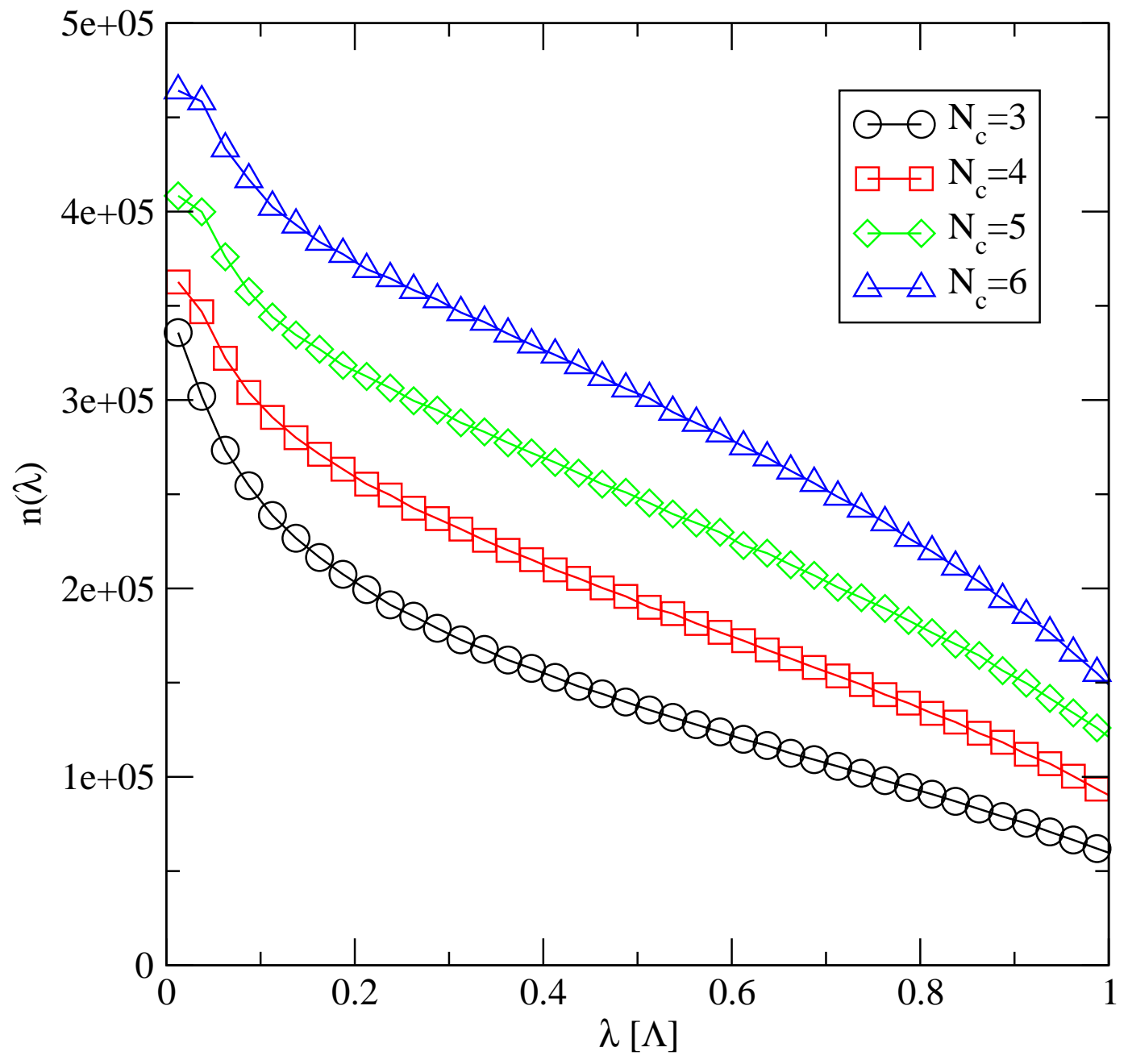

FIG. 9: Spectrum of the Dirac operator in a pure gauge instanton ensemble for different numbers of colors. The eigenvalues are given in units of the QCD scale parameter. The results were obtained using numerical simulations with $N=128$ instantons. 


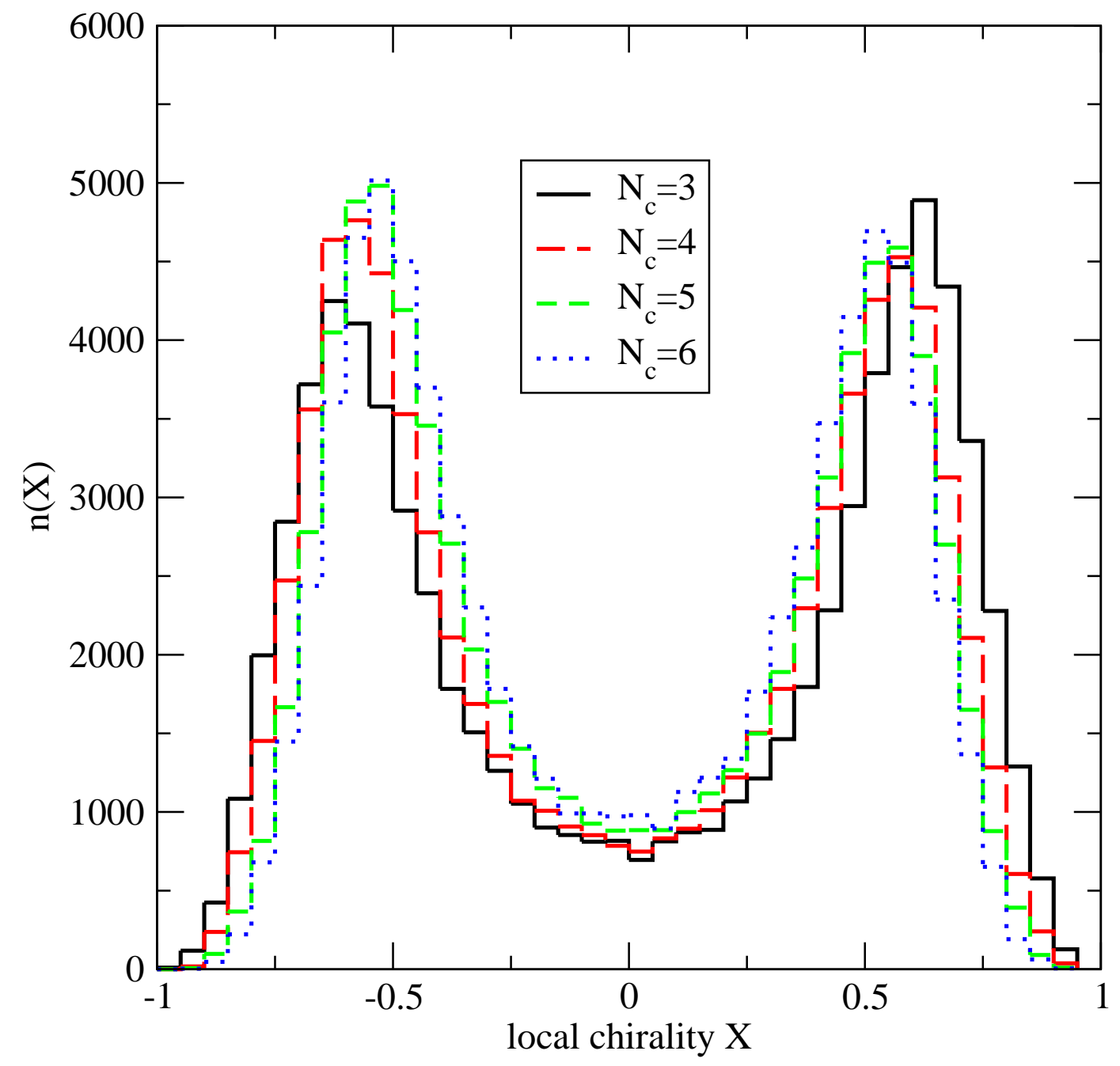

FIG. 10: Local chirality distribution function in a pure gauge instanton ensemble for different numbers of colors. The local chirality $X$ is defined in the text. The results were obtained using numerical simulations with $N=128$ instantons. 
a)

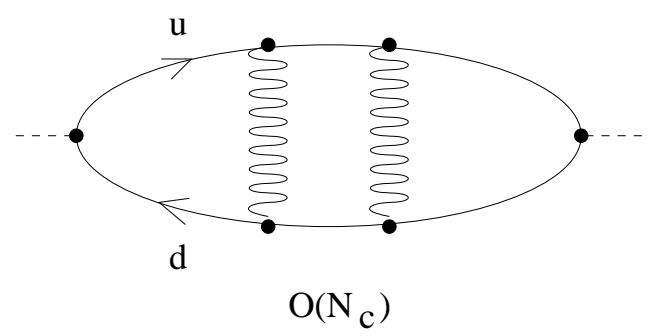

c)

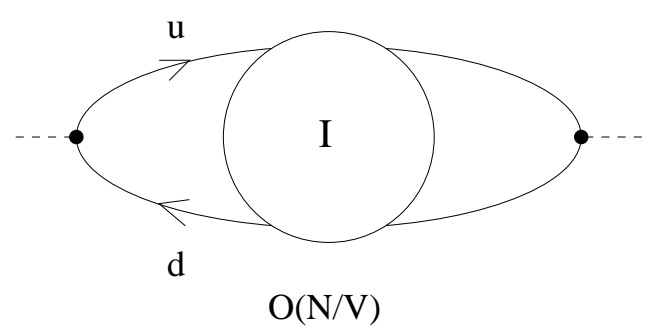

b)

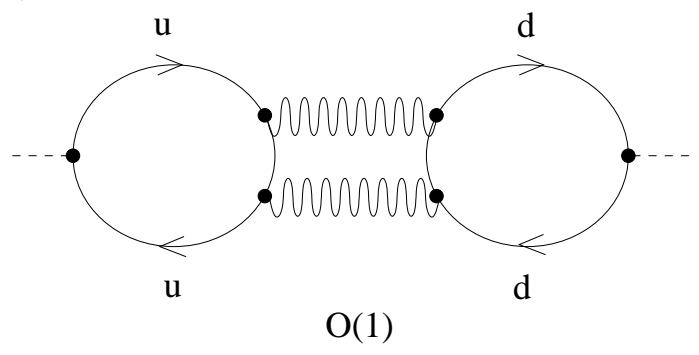

d)

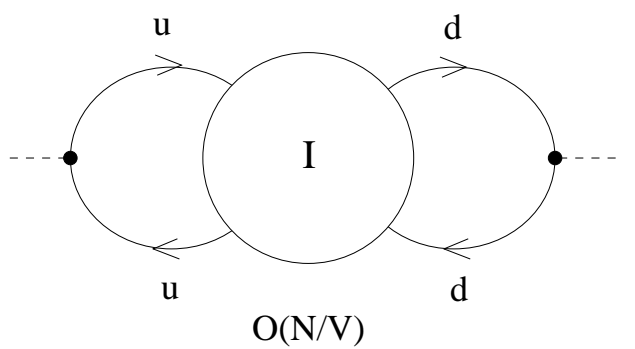

FIG. 11: Comparison of perturbative and instanton contributions to connected and disconnected correlation functions. The perturbative contribution to the disconnected correlator Fig. b is suppressed by a factor $1 / N_{c}$ compared to the connected correlator Fig. a. The single instanton contribution to the two correlation functions, shown in Figs. $\mathrm{c}$ and d, is the same, up to a sign. 


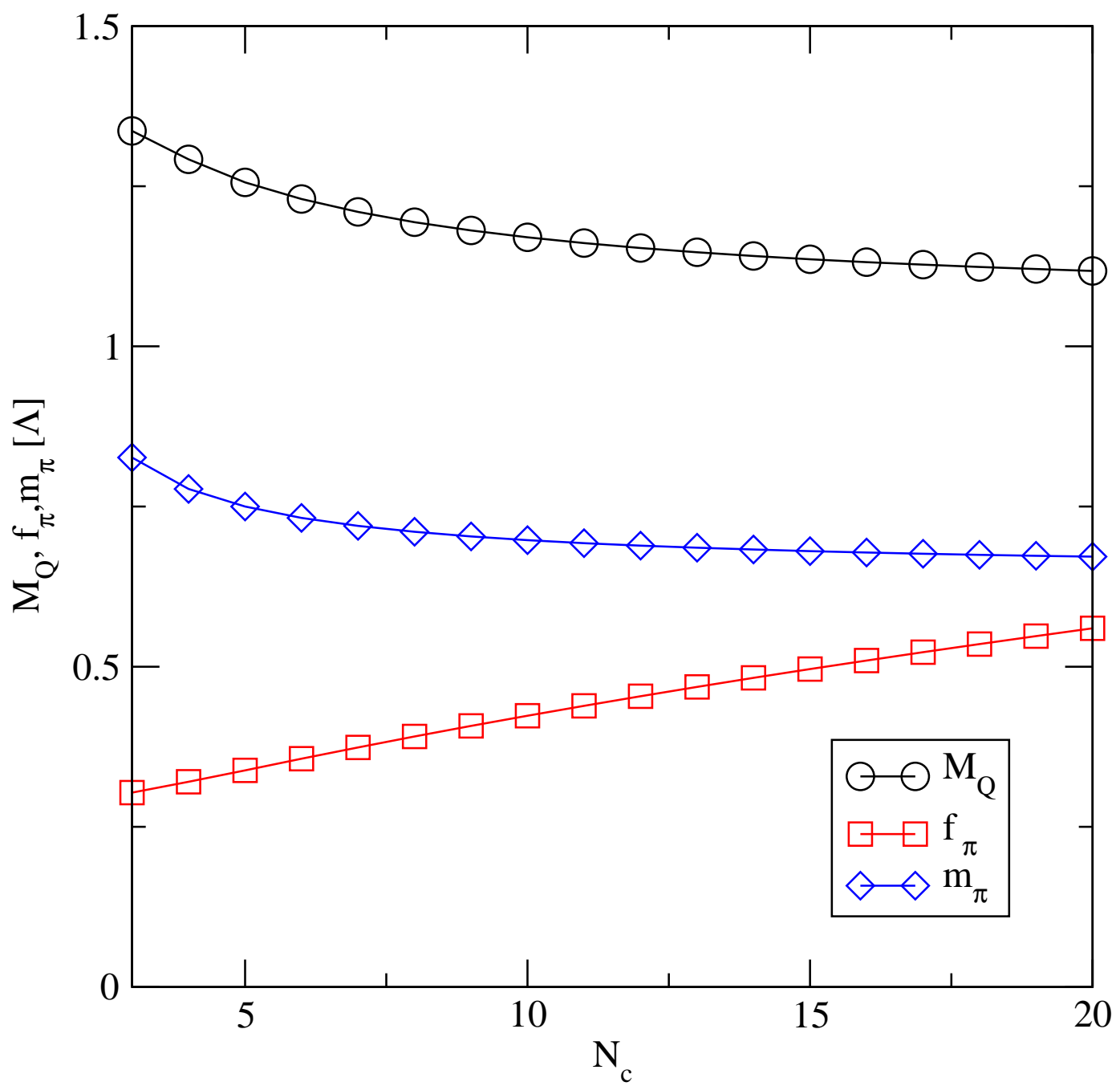

FIG. 12: Constituent quark mass $M_{Q}$, pion mass $m_{\pi}$, and pion decay constant $f_{\pi}$ as a function of the number of colors $N_{c}$. All quantities are given in units of the QCD scale parameter $\Lambda$. The results shown in this figure were obtained using the mean field approximation. 


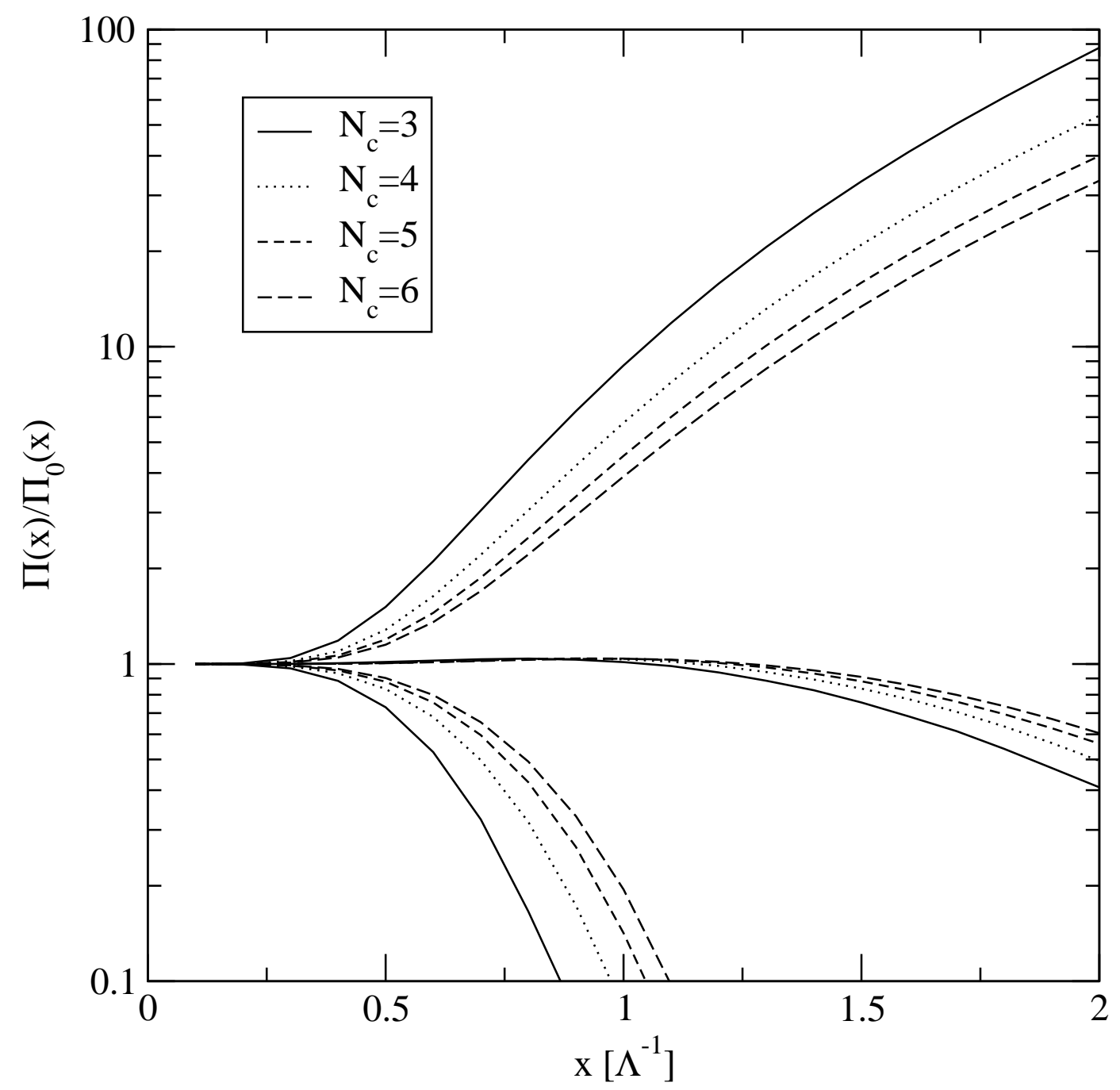

FIG. 13: Correlation functions in the pion, rho meson, and $\eta^{\prime}$ meson channel. The correlators are shown as a function of the distance in units of the inverse scale parameter. The correlation functions are normalized to free field behavior, $\Pi_{0}(x) \sim N_{c} / x^{6}$. The results shown in this figure were obtained using the mean field approximation. 


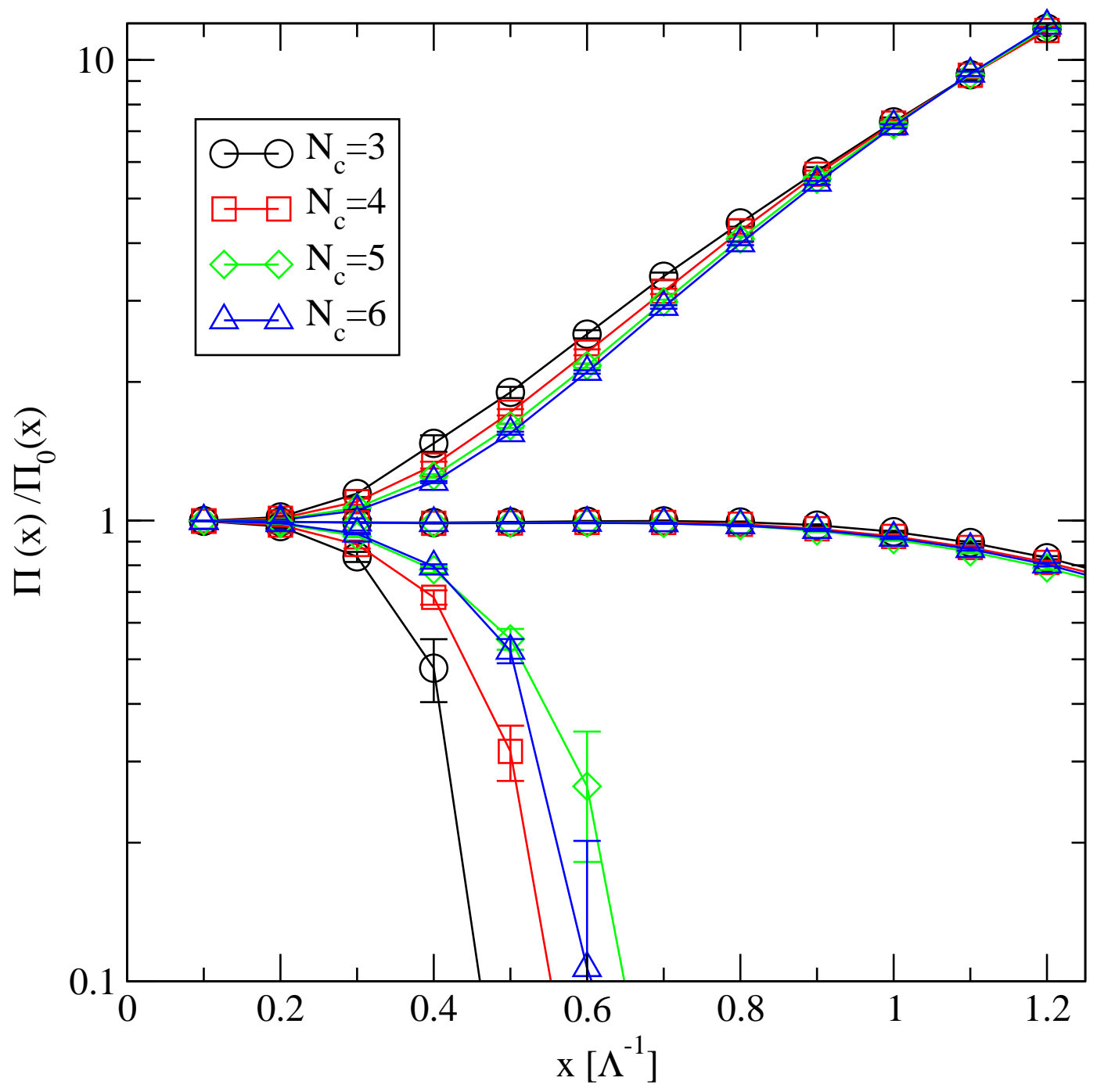

FIG. 14: Meson correlation function in a pure gauge instanton ensemble for different numbers of colors. We show the correlation function of the pion, the rho meson, and the $\eta^{\prime}$ meson normalized to the corresponding free correlation functions. 


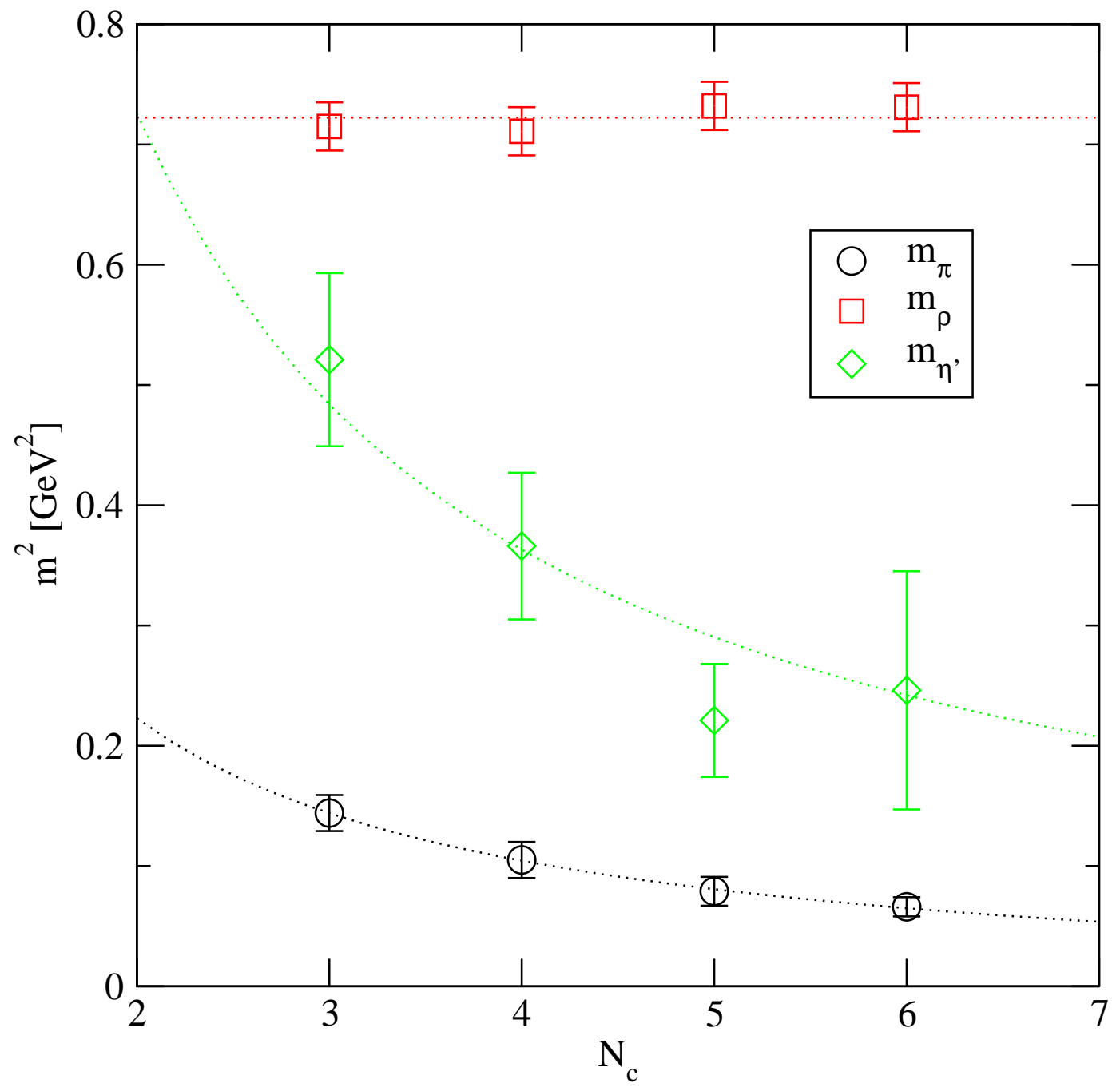

FIG. 15: Masses of the pion, the rho meson, and the $\eta^{\prime}$ meson extracted from meson correlation function in a pure gauge instanton ensemble for different numbers of colors. The results were converted to physical units using $\Lambda=0.2 \mathrm{GeV}$. The quark mass was chosen to be $m_{q}=0.2 \Lambda=40$ $\mathrm{MeV}$. The dashed lines show fits of the form $a_{1}+a_{2} / N_{c}$ (for $m_{\pi}^{2}$ and $m_{\rho}^{2}$ ) and $a_{2} / N_{c}$ (for $m_{\eta^{\prime}}^{2}$ ). 


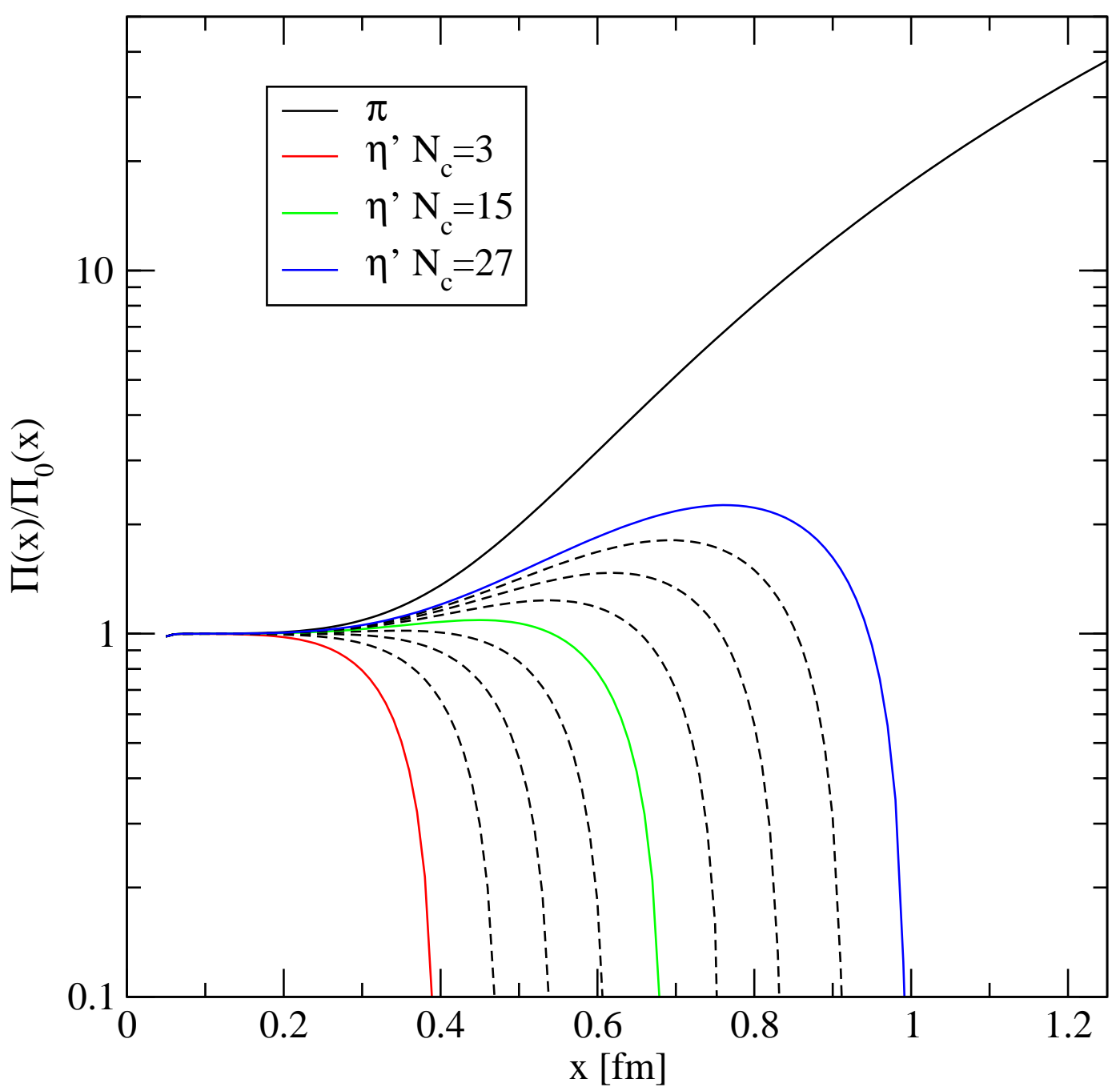

FIG. 16: Expected behavior of the quenched pion and $\eta^{\prime}$ correlation functions in the large $N_{c}$ limit. The correlation functions were computed from the spectral representation equs. (44,49) using the phenomenological values $m_{\pi}=139 \mathrm{MeV}, \lambda_{\pi}=(450 \mathrm{MeV})^{2}, E_{0}=1.3 \mathrm{GeV}$ and $m_{0}=900 \mathrm{MeV}$ $\left(N_{c}=3\right)$. We assume that $\lambda_{\pi}^{2} \sim N_{c}$ and $m_{0}^{2} \sim 1 / N_{c}$. 